Portland State University

PDXScholar

6-14-2013

\title{
A Method for Simulating Acoustic Emissions from Tensile Cracks in Sea Ice
}

Muciri Nyamu Gatimu

Portland State University

Follow this and additional works at: https://pdxscholar.library.pdx.edu/honorstheses Let us know how access to this document benefits you.

\section{Recommended Citation}

Gatimu, Muciri Nyamu, "A Method for Simulating Acoustic Emissions from Tensile Cracks in Sea Ice" (2013). University Honors Theses. Paper 21.

https://doi.org/10.15760/honors.18

This Thesis is brought to you for free and open access. It has been accepted for inclusion in University Honors Theses by an authorized administrator of PDXScholar. Please contact us if we can make this document more accessible: pdxscholar@pdx.edu. 


\section{A Method for Simulating Acoustic Emissions from Tensile Cracks in Sea Ice}

By

Muciri Nyamu Gatimu

Undergraduate Honors Thesis:

Bachelor of Science in Physics

Bachelor of Science in Geology

Advisors:

Dr. Lisa M. Zurk

Dr. Michael L. Cummings

Portland State University

(C)2013 


\section{Acknowledgments}

I would like to thank Dr. Lisa Zurk, for giving me the opportunity to study such a fascinating topic previously unknown to me. I would also like to thank John Gebbie for his patience and knowledge in addressing my questions about signal processing and OASN. Nathan Laws for the RAM simulation and much needed feedback on my thesis. To Dr. Drake C. Mitchell, for allowing me to use your lab as a home base for my rack server and annoy everyone in the lab with the cacophony of noise displaced by that graceful machine. And last but not least, the Marvin H. Beeson Undergraduate Research Award Endowment, for purchasing my rack server to aid in my simulation endeavors.

Special thanks is also given to the Scripps Oceanographic Institute for providing hydrophone data of ambient noise. 


\section{Contents}

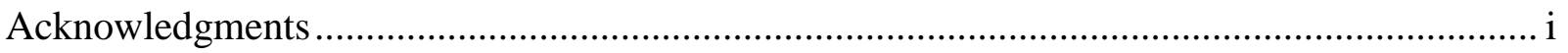

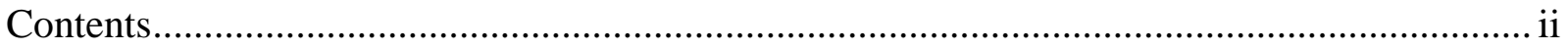

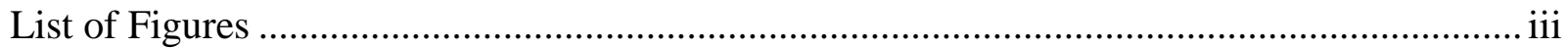

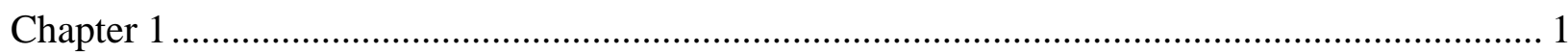

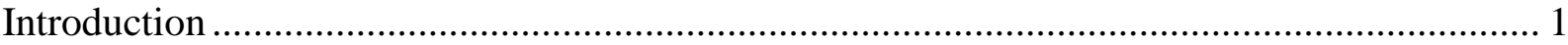

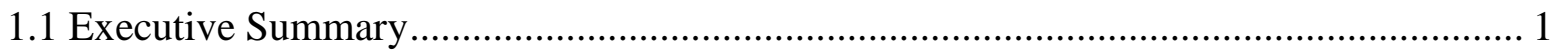

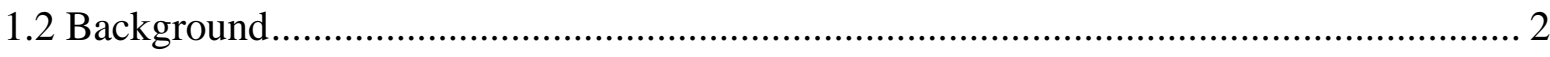

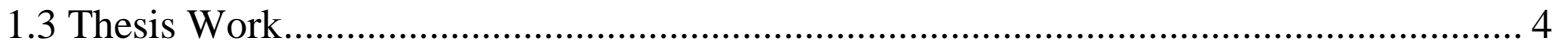

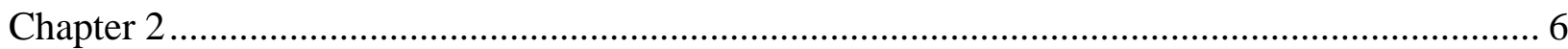

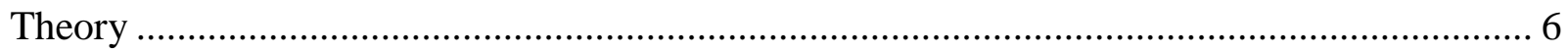

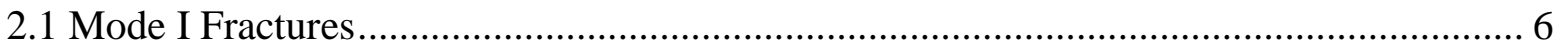

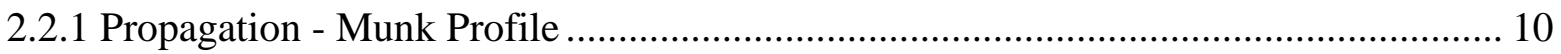

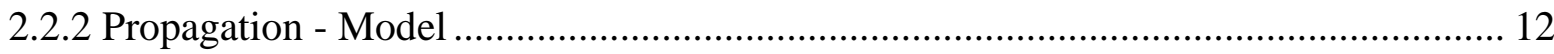

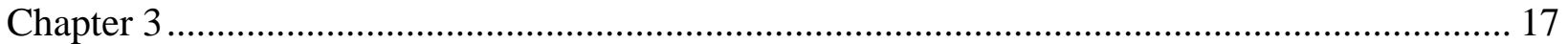

OASN: NOISE, COVARIANCE MATRICES AND SIGNAL REPLICAS ....................... 17

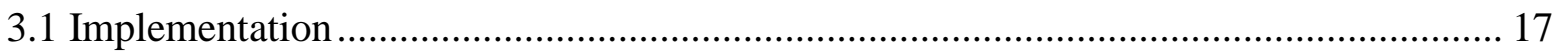

3.2 Wavenumber Integration Induced Aberrations ................................................... 18

3.3 OASN Comparison with RAM........................................................................ 26

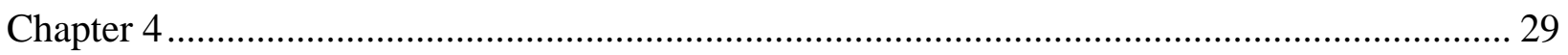

Simulation Data and Comparison to Observed Ambient Noise ............................................. 29

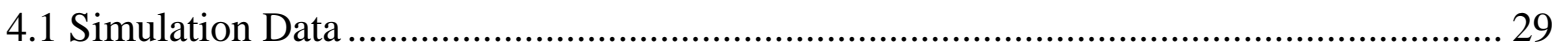

4.2 Comparison to Observed Ambient Noise .............................................................. 35

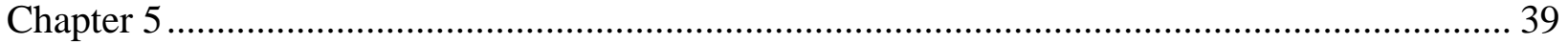

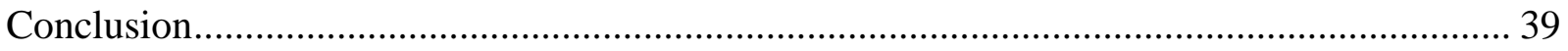

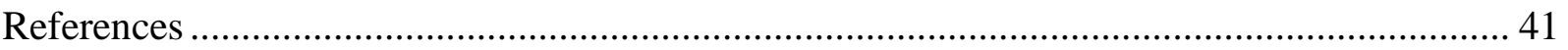




\section{List of Figures}

Figure 1.1 - Red stars represent discrete point sources whereas the yellow circle represents the hydrophone. The wavefront from the point sources are shown as red arcs.

Figure 2.1 - Initial formation of a Tensile Crack. The arrow into the page denotes the direction of crack propagation whereas the arrows pointing in separate directions perpendicular to crack propagation denote block displacement. Reprinted from B. A. Van Der Pluijm and S. Marshak, Earth Structure Second Edition p.122, New York, New York: W.W. Norton \& Company, Inc., 2004. 6

Figure 2.2 - The principle stresses on a body. The third principle stress $\sigma_{2}$ is not shown but is taken as being orthogonal to $\sigma_{1}$ and $\sigma_{3}$ and thus into the page. Reprinted from B. A. Van Der Pluijm and S. Marshak, Earth Structure Second Edition p.119, New York, New York: W.W. Norton \& Company, Inc., 2004.

Figure 2.3 - Formation of a tensile crack induced by the maximum principle stress. Smaller arrows inside block denote movement or opening of tensile crack. The third principle stress $\sigma_{2}$ is not shown but is taken as being orthogonal to $\sigma_{1}$ and $\sigma_{3}$ and thus into the page. Adapted from B. A. Van Der Pluijm and S. Marshak, Earth Structure Second Edition p.119, New York, New York: W.W. Norton \& Company, Inc., 2004.

Figure 2.4 - Method of fracture start and stop jumping applied to simulations. Arrows depict direction of fracture propagation at velocity $\mathrm{V}_{\text {jump. }}$. Red bar shows a pause between fracture jumps.

Figure 2.5 - Munk sound speed profile in deep water.

Figure 2.6 - Transmission loss in $\mathrm{dB}$ of a $100 \mathrm{~Hz}$ impulse at 100 meters depth with a Munk profile....... 12 Figure 2.7 -The red line perpendicular to the layers is the plane of incidence. The incident ray is denoted with angle $\left(\Theta_{\mathrm{I}}\right)$ to the plane of incidence whereas the reflected ray and transmitted ray are represented by angle $\left(\Theta_{R}\right)$ and angle $\left(\Theta_{T}\right)$ respectively to the plane of incidence. .13 
Figure 2.8 - Leaky waveguide or Pekeris waveguide acoustic paths between source (red star) and hydrophone (yellow circle). Ray (1) is the shortest path having two boundary interactions. Ray (2) is the second shortest path having only three boundary interactions. The red ray (3) either leaked out of the waveguide or was transmitted through the ice to eventually become incident upon the ice again due to the Munk profile. 15

Figure 2.9 -Waterborne acoustic paths between source (red star) and hydrophone (yellow circle). Ray (1) is the shortest path with one boundary interaction. Ray (2) is the second shortest path with two boundary interactions. Ray (3) is the longest path with three boundary interactions. .16

Figure 3.1 - Geometry for all simulations conducted in this study. Red stars represent discrete point sources whereas the yellow circle represents the hydrophone. The wavefront from the point sources are shown as red arcs.

Figure 3.2 - Simulated time series with an aberration located around $0.2 \mathrm{~s}$

Figure 3.3 - Spectrum of the simulated time series in Figure 3.2 with an aberration located around 0.2 seconds comprised of the full bandwidth of frequency for the simulation.

Figure 3.4 - Simulated time series with the aberration removed. The geometry of the simulation is identical to that in Figure 3.2.

Figure 3.5 - Spectrogram of the simulated time series in Figure 3.4. The geometry of the simulation is identical to that of Figure 3.2. .25

Figure 3.6 - Simulated Sound Pressure Level (SPL) comparison of identical geometry between OASN with aberration (Red) and OASN without aberration (blue). 26

Figure 3.7 - Simulated time series comparison between OASN with aberration reduced (blue) and RAM (red) for a single source within sea ice. 27

Figure 3.8 - Simulated Sound Pressure Level (SPL) comparison between OASN with aberration reduced (Blue) and RAM (red). The geometry of the simulation is identical to that of Figure 3.7. .27

Figure 4.1 - Simulated time series of a tensile crack propagating toward the hydrophone at $550 \mathrm{~m} / \mathrm{s} . \ldots . .30$ 
Figure 4.2 - Spectrogram of the simulated time series shown in Figure 4.1 ........................................ 30

Figure 4.3 - Simulated time series of a tensile crack propagating toward the hydrophone at $400 \mathrm{~m} / \mathrm{s} . \ldots . .31$

Figure 4.4 - Spectrogram of the simulated time series shown in Figure 4.3 ........................................ 31

Figure 4.5 - Simulated time series of a shallow tensile crack propagating toward the hydrophone at 285

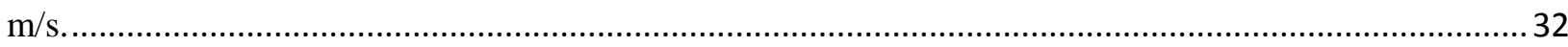

Figure 4.6 - Spectrogram of the simulated time series shown in Figure 4.5 ......................................... 32

Figure 4.7 - Simulated time series of a deep tensile crack propagating toward the hydrophone at $285 \mathrm{~m} / \mathrm{s}$.

Figure 4.8 - Spectrogram of the simulated time series shown in Figure 4.7 ....................................... 33

Figure 4.9 - Observed time series of sea ice deformation event one .................................................... 36

Figure 4.10 - Observed spectrogram of time series for sea ice deformation event one in Figure 4.9........36

Figure 4.11 - Observed time series of sea ice deformation event two................................................ 37

Figure 4.12 - Observed spectrogram of time series for sea ice deformation event one in Figure 4.11......37 


\section{Chapter 1}

\section{Introduction}

\subsection{Executive Summary}

As the seasons change, sea ice in the polar regions undergoes a freeze-thaw cycle producing anisotropic sea ice in terms of physical properties and structures [1] [2]. Deformation of these variable structures from the freeze-thaw cycle and flux of energy within the polar regions causes the ice to fracture radiating noise into the water [3] [4] [5] [6]. Simulating the noise from sea ice is difficult due to the variability in the physical properties of sea ice, dynamic environment of the polar regions, complex physics governing sound propagation and numerous methods by which the emission of noise can be generated from sea ice [7] [8] [9]. The radiated ambient noise generated from deforming sea ice can influence the habits of oceanic life, targeting abilities of vessels equipped with sonar, and add to the background acoustic noise in the polar regions.

This work addresses the noise generated by opening (Mode I) fractures in sea ice. OASN [10] was used to simulate acoustic waves propagating though the complex arctic environment. For this problem, a significant understanding of mathematics was required to overcome a mathematical aberration, within the computational tool, which was reduced and compared against simulations computed by an alternate propagation tool, RAM [11]. Simulations of Model I fractures were compared against observed data. The methods developed for the simulation of Mode I fractures was proven successful. 


\subsection{Background}

The Arctic and Antarctic regions of the Earth are known for vast areas of fluctuating sea ice. It has been known for some time that noise, or the release of energy, is present around melting icebergs [1] [2]. As the seasons change, sea ice undergoes a cycle of freezing and thawing which is further complicated by electromagnetic and gravitational forces. The melting and deformation of sea ice causes the ice to fracture, producing a fluctuating range of noise that radiates into the surrounding water. The acoustic emissions from sea ice radiate into the water and contribute to the ambient noise of the polar regions [5] [4] [9]. This radiative noise can influence the habits of oceanic life, affect abilities of vessels equipped with sonar, and add to the background acoustic noise in the polar regions.

The ice fractures that emit sea ice noise are produced by the dynamic environments of the polar regions. As sea ice ages, its physical properties, and thus its interaction with the environment, also change accordingly [7] [8] [6]. The sea ice becomes more dense due to the prolonged exposure to the Earth's gravitation field, causing the interstitial spaces between crystal structures to shrink in magnitude. As the sea ice deforms, fractures can irrupt due to the heterogeneity, but more consistently fractures result from the deformation [3]. Fractures resulting from deformation are common in the formation of pressure ridges and the movement of sea ice along and against each other. Three types of fractures exist: Mode I (opening), Mode II (shearing) and Mode III (tearing). Each of these fractures propagate in a jumping fashion causing variations in the velocity of a propagating crack [12] [13] [14]. This study address the acoustic emissions from Mode I fractures in sea ice. 


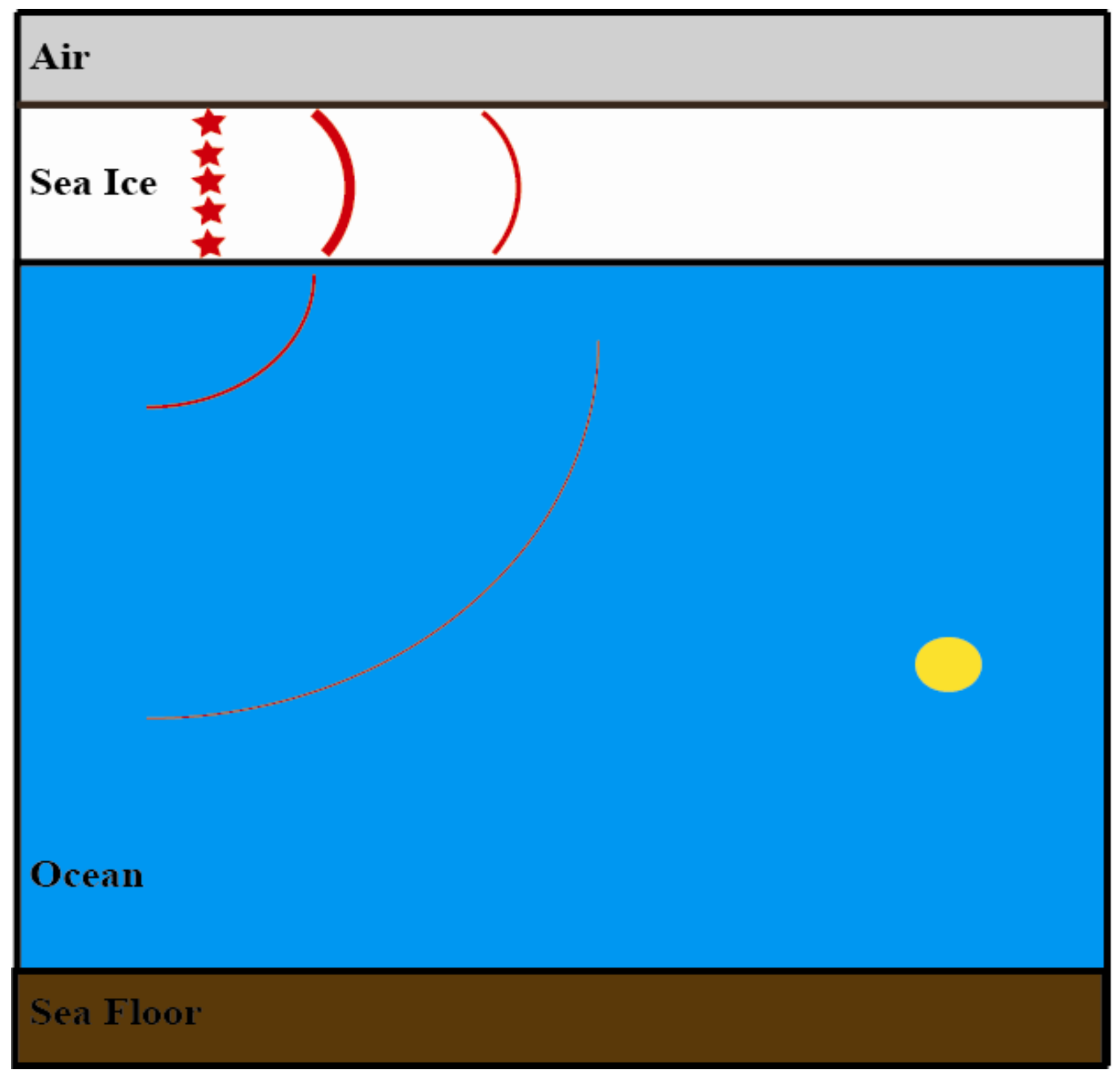

Figure 1.1 - Red stars represent discrete point sources whereas the yellow circle represents the hydrophone. The wavefront from the point sources are shown as red arcs.

It has been shown that propagating fractures in sea ice can be discretized into a moving monopole point source [15]. Expanding upon the monopole source, discrete point sources were used to simulate a propagating Mode I fracture through the sea ice. Figure 1.1 shows a Mode I fracture, at an instant in time, in sea ice as discretized point sources. The sea ice sandwiched 
between the air and water forms a leaky waveguide, allowing acoustic waves to propagate along two main paths, either through the leaky waveguide or through the water. The decrease in sound pressure level as the wavefront propagates is depicted as a thinner arc. In this study, the sea ice is modeled as a homogenous, laminar, perfectly smooth structure with uniform thickness and constant sound speed.

\subsection{Thesis Work}

This thesis addressed the problem of acoustic emissions from sea ice by investigating ambient noise emitted by Mode I fractures caused by the deformation of sea ice. Mode I fractures were simulated by discretizing the acoustic wave of the fracture into point sources within the sea ice. The inherent jumping characteristic during propagation of Mode I fractures was taken into account by the incorporation of pause increments based on the range of fracture propagation. The acoustic propagation was simulated as a waveguide structure formed by the air, sea ice and water column.

In Summation:

- Addressed the major causes of ambient noise from sea ice in the polar regions.

- Developed a model to simulate the ambient noise from Mode I fractures of sea ice in the polar regions.

- Reduced aberration in OASN allowing modeling of Mode I fractures in sea ice.

- Verified OASN implementation by comparison with simulations computed with an alternative modeling tool, RAM. 
- Compared OASN simulations to observed ambient noise data from the arctic regions.

- Found several analogous characteristics between simulated and observed data, which suggest that the methods developed are favorable in further investigations of ambient noise from sea ice deformation. 


\section{Chapter 2}

\section{Theory}

\subsection{Mode I Fractures}

Mode I fractures, Figure 2.1, or more commonly referred to as opening fractures, tensilemode cracks or tensile cracks, are the source of ambient noise considered in this thesis. To understand what is technically defined as a tensile, crack the concept of principle stresses $(\sigma)$ must first be explained. In fracture mechanics, the forces present upon a body are separated into three orthogonal axes, similar to Cartesian coordinates, called the principal stresses.

\section{Tensile-mode cracks}

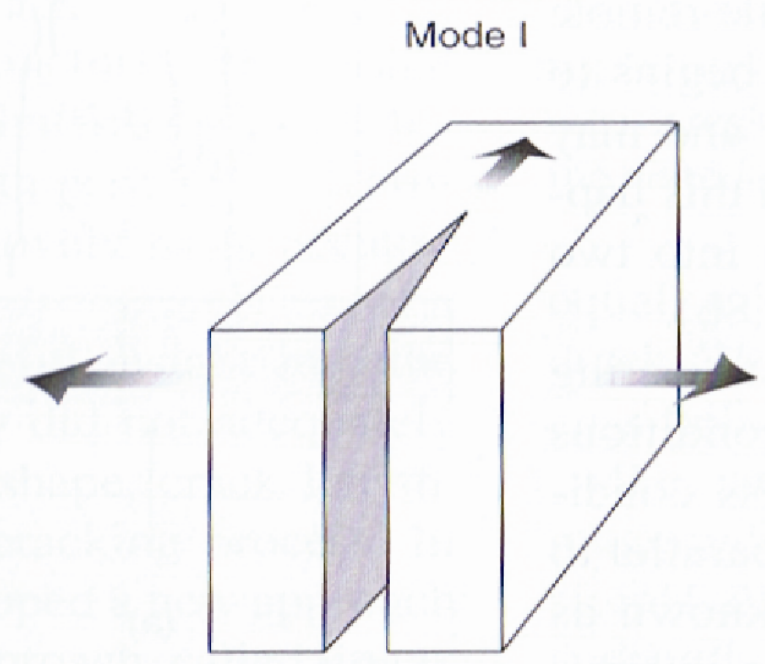

Figure 2.1 - Initial formation of a Tensile Crack. The arrow into the page denotes the direction of crack propagation whereas the arrows pointing in separate directions perpendicular to crack propagation denote block displacement. Reprinted from B. A. Van Der Pluijm and S. Marshak, Earth Structure Second Edition p.122, New York, New York: W.W. Norton \& Company, Inc., 2004.

Each of these principal stresses represents the stress or force per unit area (units of pressure) on a given body. If the principal stresses are each of varying magnitude, then the principal stress with the largest magnitude is denoted by $\sigma_{1}$. The second and third principle stresses, ordered by 
magnitude, are represented by $\sigma_{2}$ and $\sigma_{3}$, respectively. Figure 2.2 shows the principal stresses on an arbitrary body.

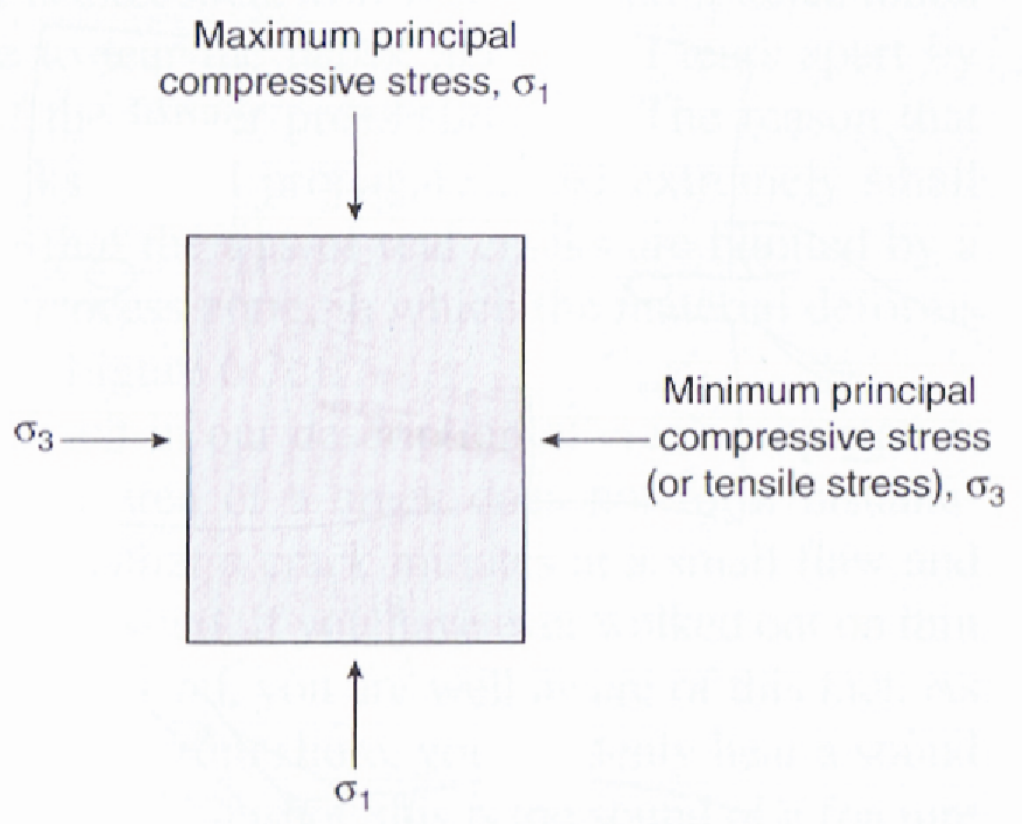

Figure 2.2 - The principle stresses on a body. The third principle stress $\sigma_{2}$ is not shown but is taken as being orthogonal to $\sigma_{1}$ and $\sigma_{3}$ and thus into the page. Reprinted from B. A. Van Der Pluijm and S. Marshak, Earth Structure Second Edition p.119, New York, New York: W.W. Norton \& Company, Inc., 2004.

A tensile crack is a type of fracture with the propagation of the crack perpendicular to the applied maximum principle stress $\sigma_{1}$. Figure 2.3 shows an example of an opening crack with principal stresses $\sigma_{2}$ and $\sigma_{2}$ being of equal magnitude. There are many methods of creating opening cracks. The method that is chosen, however, is not critical in this study, as the opening cracks within the sea ice are taken as a wavefront from the energy released by the breaking of bonds within the sea ice. Some of the energy released in breaking the bonds is subsequently emitted in the form of acoustic energy. 


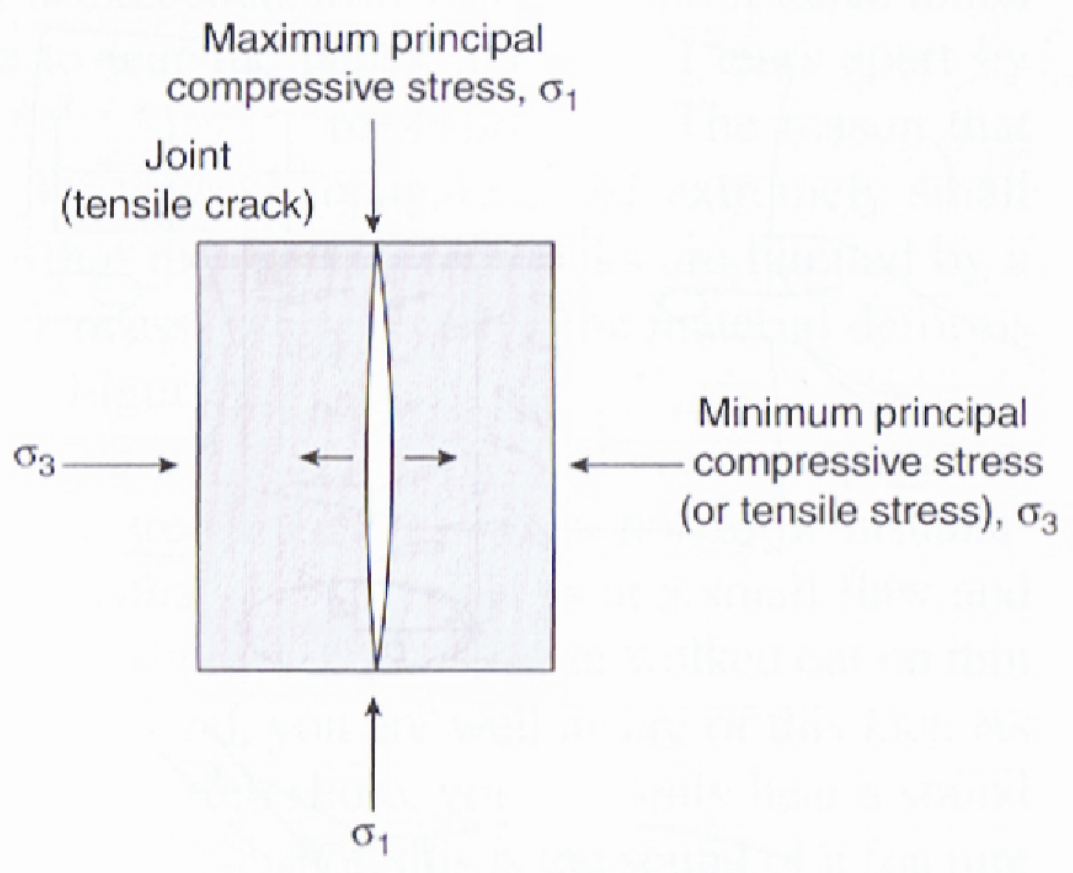

Figure 2.3 - Formation of a tensile crack induced by the maximum principle stress. Smaller arrows inside block denote movement or opening of tensile crack. The third principle stress $\sigma_{2}$ is not shown but is taken as being orthogonal to $\sigma_{1}$ and $\sigma_{3}$ and thus into the page. Adapted from B. A. Van Der Pluijm and S. Marshak, Earth Structure Second Edition p.119, New York, New York: W.W. Norton \& Company, Inc., 2004.

As a tensile crack propagates, the leading edge produces a wavefront that propagates into the environment.

During the propagation of a tensile crack, its velocity varies according to two methods of propagation. The first method, which was applied in this study, is a start and stop pattern, with brief moments of complete rest, show in Figure 2.4. The second method consists of periods of positive and negative acceleration similar to the first method, without coming to a complete stop. Instead of propagating with a linear velocity - and thus a null acceleration - tensile cracks propagate in a jumping fashion, accelerating to a high velocity 
Fracture Start

Fracture Pause

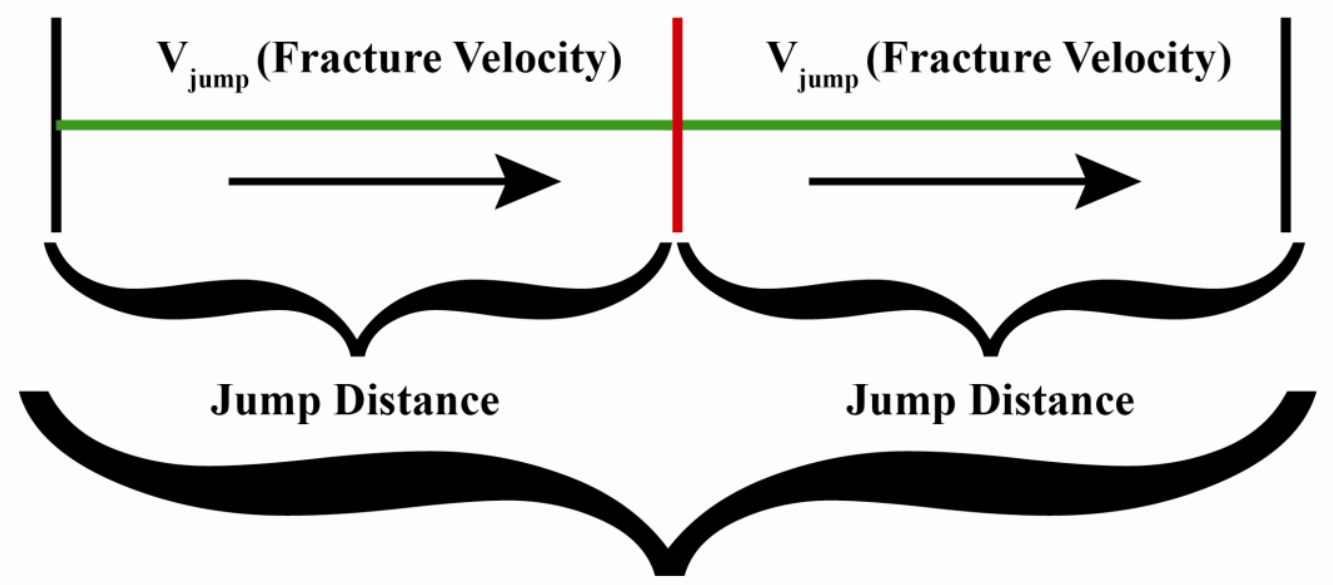

Fracture Length

Figure 2.4 - Method of fracture start and stop jumping applied to simulations. Arrows depict direction of fracture propagation at velocity $V_{\text {jump. }}$. Red bar shows a pause between fracture jumps.

within a short period of time to arrest or slowdown for a brief duration before accelerating again

[13]. The propagating crack velocity or the fracture velocity of jumps $\left(\mathrm{V}_{\text {jump }}\right)$ varies from $0-550$

$\mathrm{m} / \mathrm{s}$ [13] [14] [12]; the average velocity of a tensile crack during periods of slowing down ranges

from 0-300 m/s [13] [14]. To simulate pauses, a jump distance (units of meters) and a fracture

pause (units of seconds) was specified. Fracture pauses were placed at intervals of the jump

distance along the fracture length. As the propagating fracture reached a fracture pause, it was

held at that distance for the duration of the fracture pause before continuing to propagate.

In order to simulate a propagating wavefront, discretized point sources were used to

recreate the wavefront produced by a propagating tensile crack. The point source,

$$
\Psi(\mathbf{r})=\mathbf{A} \frac{\mathrm{e}^{\mathrm{i} k \mathbf{r}}}{\mathbf{r}}
$$

, is an omnidirectional propagating wave. 
The displacement of the propagating spherical sound wave $(\psi)$ is a function of the radial distance from its center ( $\mathrm{r}$ ) for a functional dependent wavenumber (k) with specified amplitude (A) of the spherical wave. Point sources were placed in a grid with the dimensions of the fracture length and depth. Each point source was separated at a distance smaller than the spatial Nyquist. To decrease simulation time, each point source within the grid was simulated individually and thus there was no interference between point sources. A wavefront was compiled by adding the simulated outputs for an entire array of point sources - a linear arrangement of sources through the depth of the fracture. To simulate a propagating tensile crack, each array of point sources was added with a time delay that corresponded to the arrays distance away from the fracture start divided by the fracture velocity $\left(\mathrm{V}_{\text {jump }}\right)$ with the addition of any fracture pauses.

\subsubsection{Propagation - Munk Profile}

Ambient noise can be detected kilometers away from the source due to the thermodynamics of the underwater environment. The water column below the sea ice or halfspace is not homogenous in terms of physical properties. The bulk modulus $(\mathrm{K})$, density $(\rho)$ and therefore, compressional sound speed (C),

$$
C=\sqrt{\frac{K}{\rho}}
$$

, change throughout the half-space. Bulk modulus, along with density, is influenced by temperature, salinity and pressure. The salinity and pressure of the half-space increase with depth due to gravitational forces, whereas the temperature of the half-space decreases due to the penetration depth of the heat intensity throughout the half-space dropping off with the square of 
the heat energy. The rate at which the salinity, pressure and temperature change as a function of depth within the half-space varies respectively. The rate variation causes the compressional sound speed to decrease initially with depth into the half-space, eventually increasing as the varying rates of salinity, pressure and temperature affect the bulk modulus and density accordingly. This decreasing to increasing sound speed profile, called a Munk profile (Figure 2.5), causes an upward refracting index of the compressional waves.

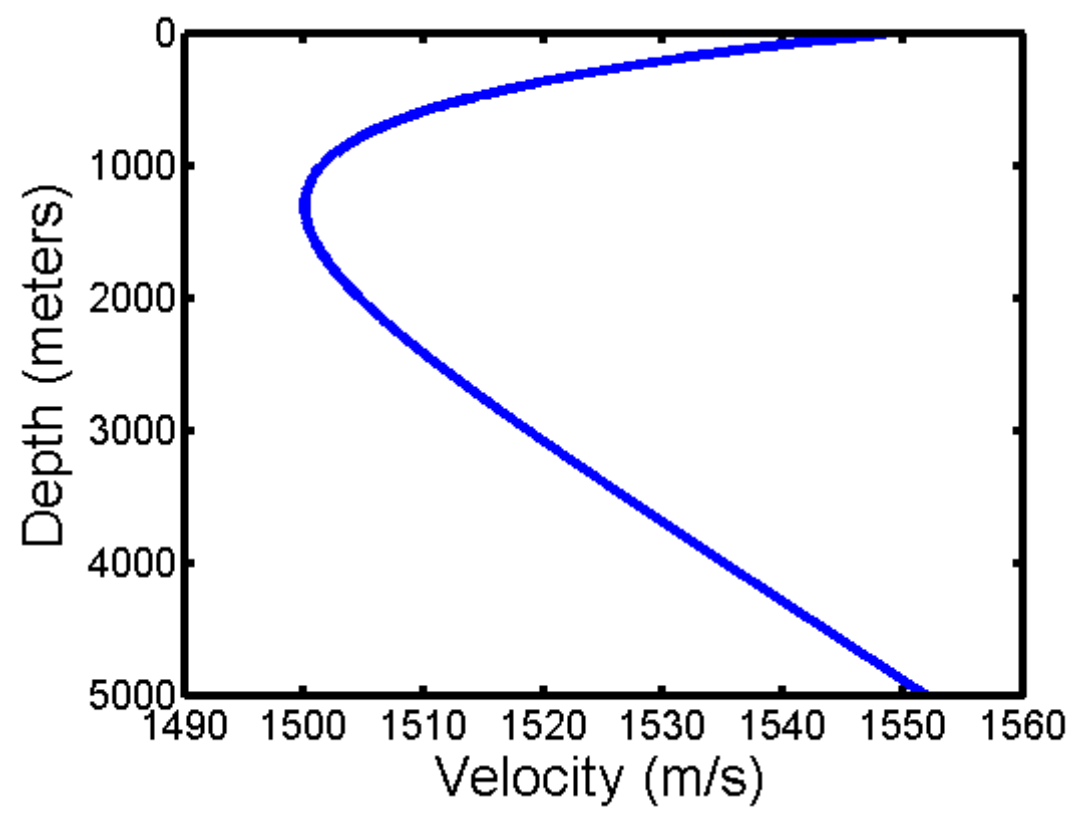

Figure 2.5 - Munk sound speed profile in deep water. 


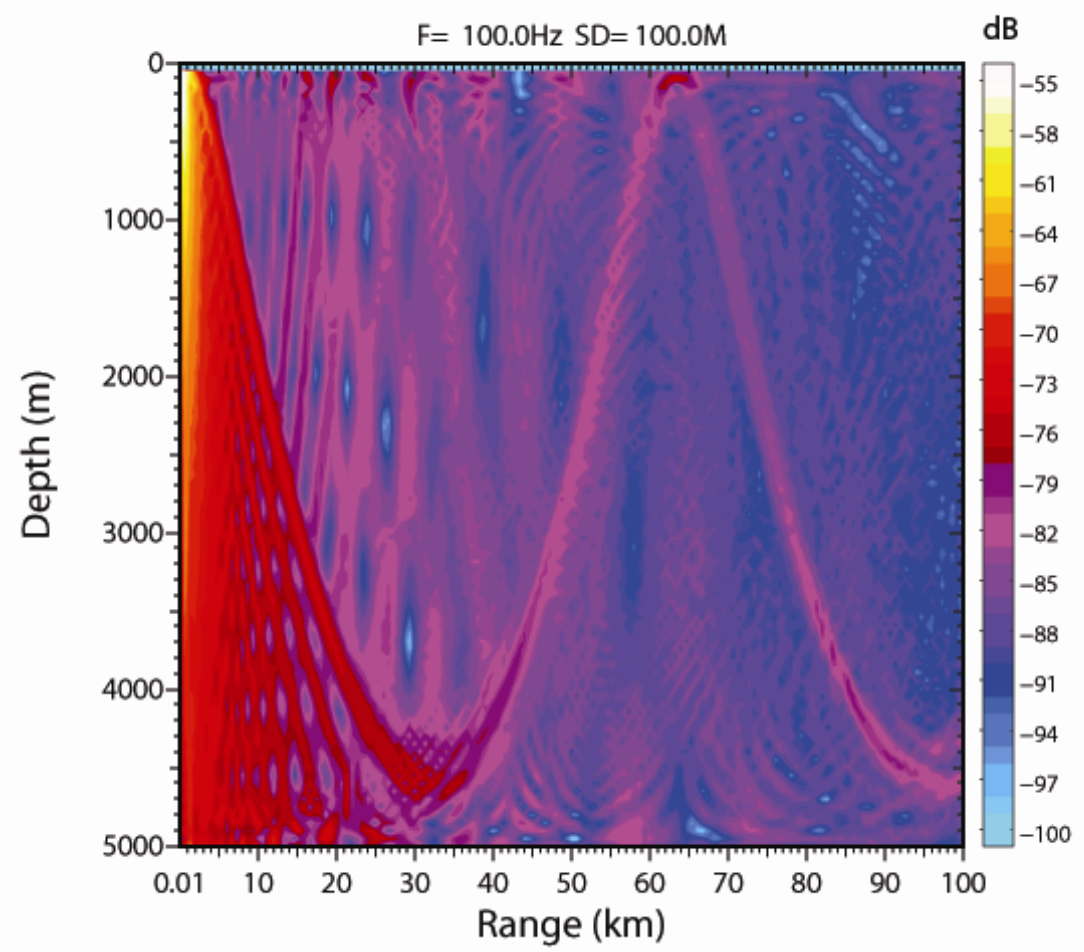

Figure 2.6 - Transmission loss in $\mathrm{dB}$ of a $100 \mathrm{~Hz}$ impulse at 100 meters depth with a Munk profile.

As compressional waves are emitted from a source near the surface, the waves refract downward toward the layer with a higher index of refraction until the compressional sound speed increases, thus causing the compressional waves to refract upward back toward the surface (Figure 2.6). These oscillatory compressional sound waves can propagate for hundreds of kilometers in deep water. Complex sound speed profiles of the polar regions only contribute to ambient noise, as sources hundreds of kilometers away can be heard as a cacophony.

\subsubsection{Propagation - Model}

The propagation of sound originating from ice fractures is bounded by the sea ice and leaks into the water forming a Pekeris waveguide or leaky waveguide [16] [3] [15]. The sound leaking from the sea ice and being transmitted to the water is shown in Figure 2.7. 


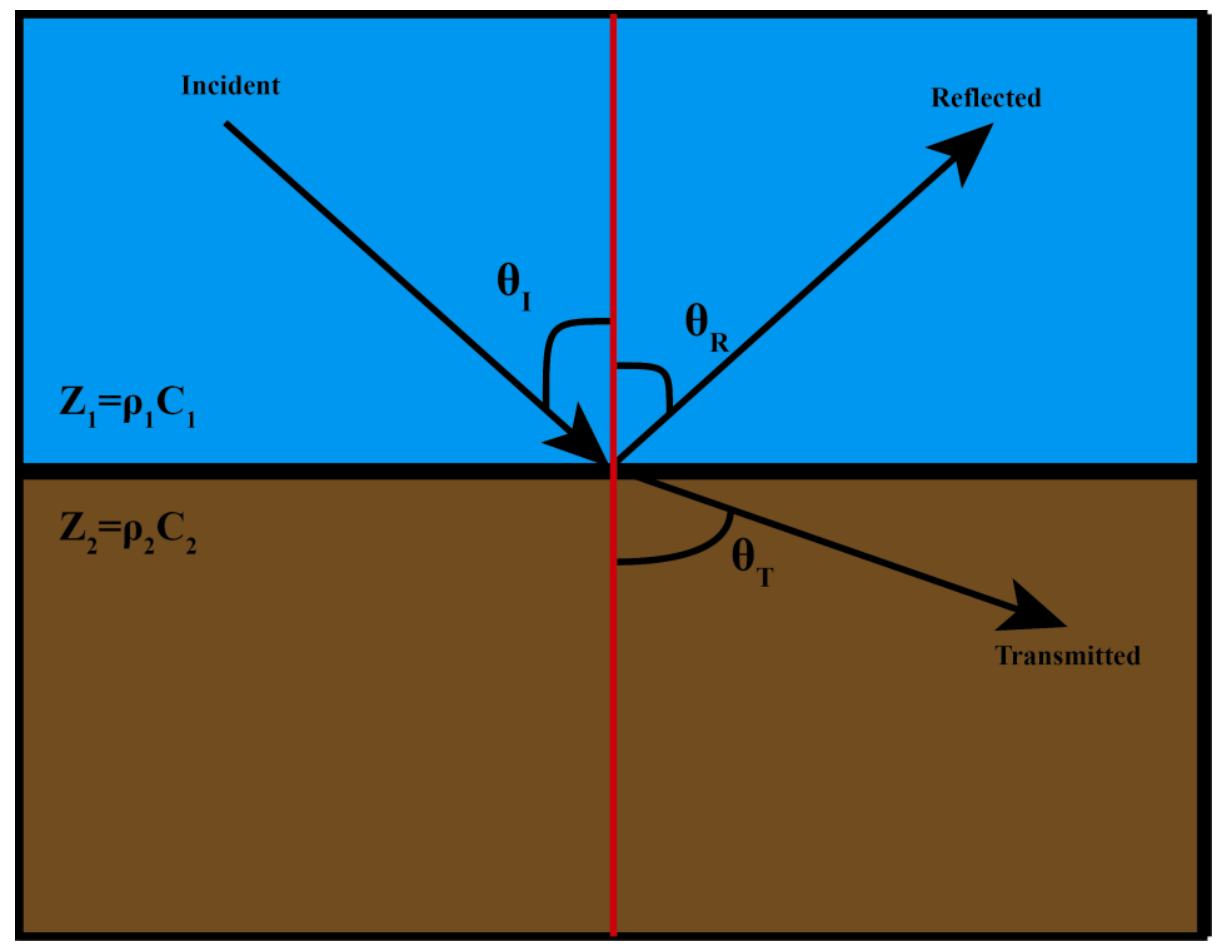

Figure 2.7 -The red line perpendicular to the layers is the plane of incidence. The incident ray is denoted with angle $\left(\Theta_{\mathrm{I}}\right)$ to the plane of incidence whereas the reflected ray and transmitted ray are represented by angle $\left(\Theta_{R}\right)$ and angle $\left(\Theta_{T}\right)$ respectively to the plane of incidence.

Figure 2.7 is a ray representation of the physics of refraction where each ray represents a spherical wave. The angle between the incident wave and the reflected wave is always the same, whereas the angle of the transmitted wave depends on the wavenumber of the medium, a relationship shown by Snell's Law,

$$
\lambda_{2} \sin \theta_{\mathrm{I}}=\lambda_{1} \sin \theta_{\mathrm{T}} .
$$

The amounts of energy transmitted and reflected depend on the acoustic impedance and angle of incidence. The acoustic impedance $(Z)$ is the ratio of acoustic pressure to the volume velocity, but can also be written in the form, 


$$
\mathrm{Z}=\rho \mathrm{C}
$$

,with impedance $(\mathrm{Z})$ being the multiplicative of the density $(\rho)$ and the phase velocity $(\mathrm{C})$ of the wave. The portions reflected and transmitted are then,

$$
\begin{aligned}
& \mathbf{R}=\frac{\mathrm{z}_{1} \cos \theta_{\mathrm{I}}-\mathrm{z}_{2} \cos \theta_{\mathrm{T}}}{\mathrm{z}_{1} \cos \theta_{\mathrm{I}}+\mathrm{Z}_{2} \cos \theta_{\mathrm{T}}}, \\
& \mathbf{T}=\frac{2 \mathrm{Z}_{2} \cos \theta_{\mathrm{I}}}{\mathrm{z}_{1} \cos \theta_{\mathrm{I}}+\mathrm{Z}_{2} \cos \theta_{\mathrm{T}}}
\end{aligned}
$$

, where $\mathrm{R}$ is the reflected and $\mathrm{T}$ is the transmitted.

The omnidirectional nature of a spherical wave, allows for many paths of propagation to take place within a specified geometry. The thickness of the sea ice and sea floor can vary along with the depth of the ocean sandwiched between them, but the main paths of propagation for each ray are still present. As the spherical waves propagate from the sea ice, there are two main path-groupings that the spherically propagating acoustic waves can take. The first path-grouping, comprised of high frequency content, due to the cutoff frequency, propagates through the sea ice and leaks from the Pekeris waveguide before reaching the hydrophone, shown in Figure 2.8. The second path-grouping is waterborne, shown in Figure 2.9, which immediately leaks out of the waveguide or is transmitted through the sea ice to the water, and propagating through the halfspace, for the majority of the duration from emission to being received at the hydrophone. The cutoff frequency $\left(\mathrm{f}_{\text {cutoff }}\right)$,

$$
f_{\text {cutoff }}=\frac{C}{2}\left(\frac{m}{B}\right)
$$


, for the leaky waveguide, where (m) is the mode - a integer from 1 to infinity - and B is the thickness of the sea ice, is an inherence characteristic of all waveguides limiting the wavelength allowed to propagate within the dimensions of the waveguide.

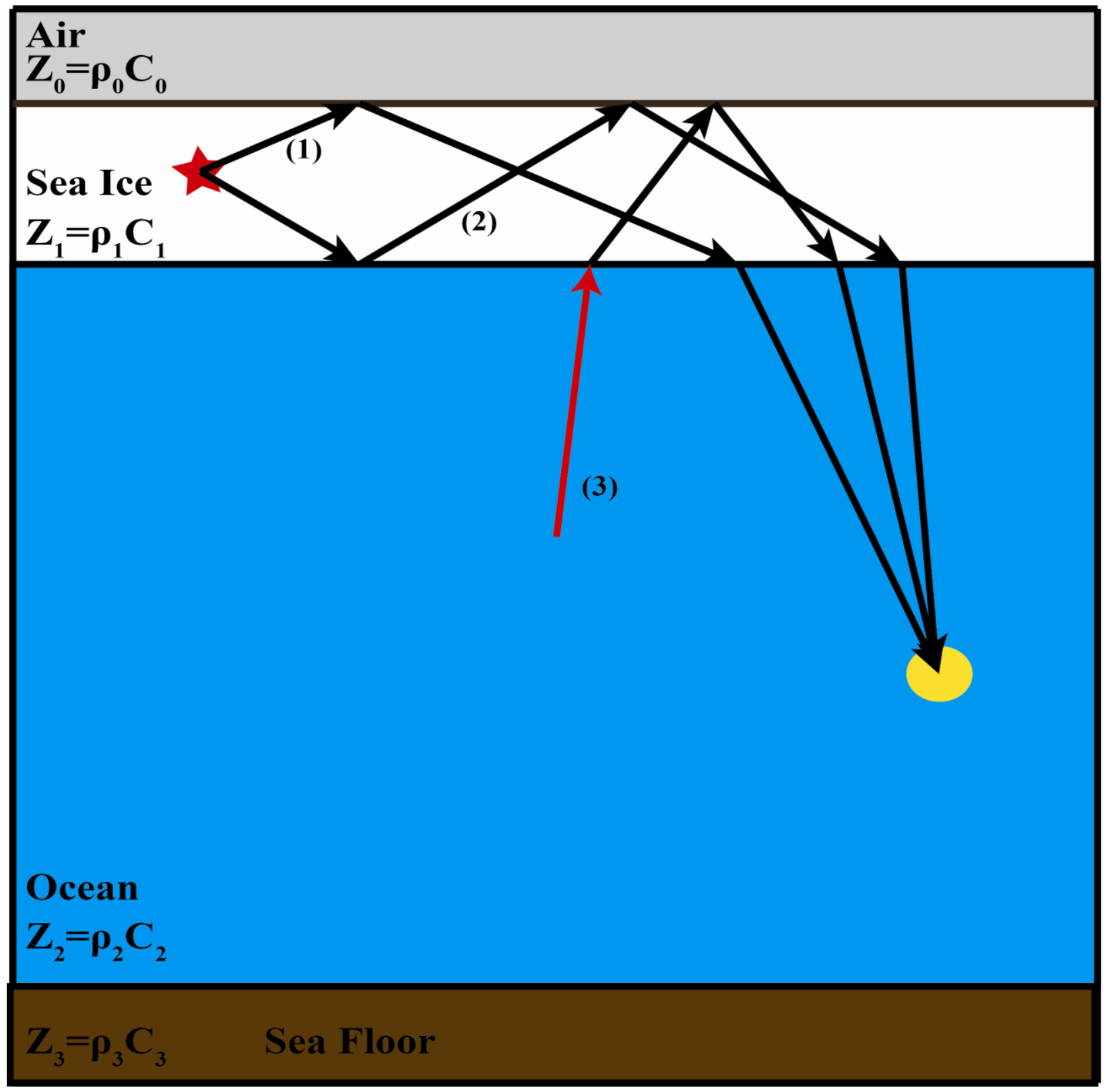

Figure 2.8 - Leaky waveguide or Pekeris waveguide acoustic paths between source (red star) and hydrophone (yellow circle). Ray (1) is the shortest path having two boundary interactions. Ray (2) is the second shortest path having only three boundary interactions. The red ray (3) either leaked out of the waveguide or was transmitted through the ice to eventually become incident upon the ice again due to the Munk profile. 


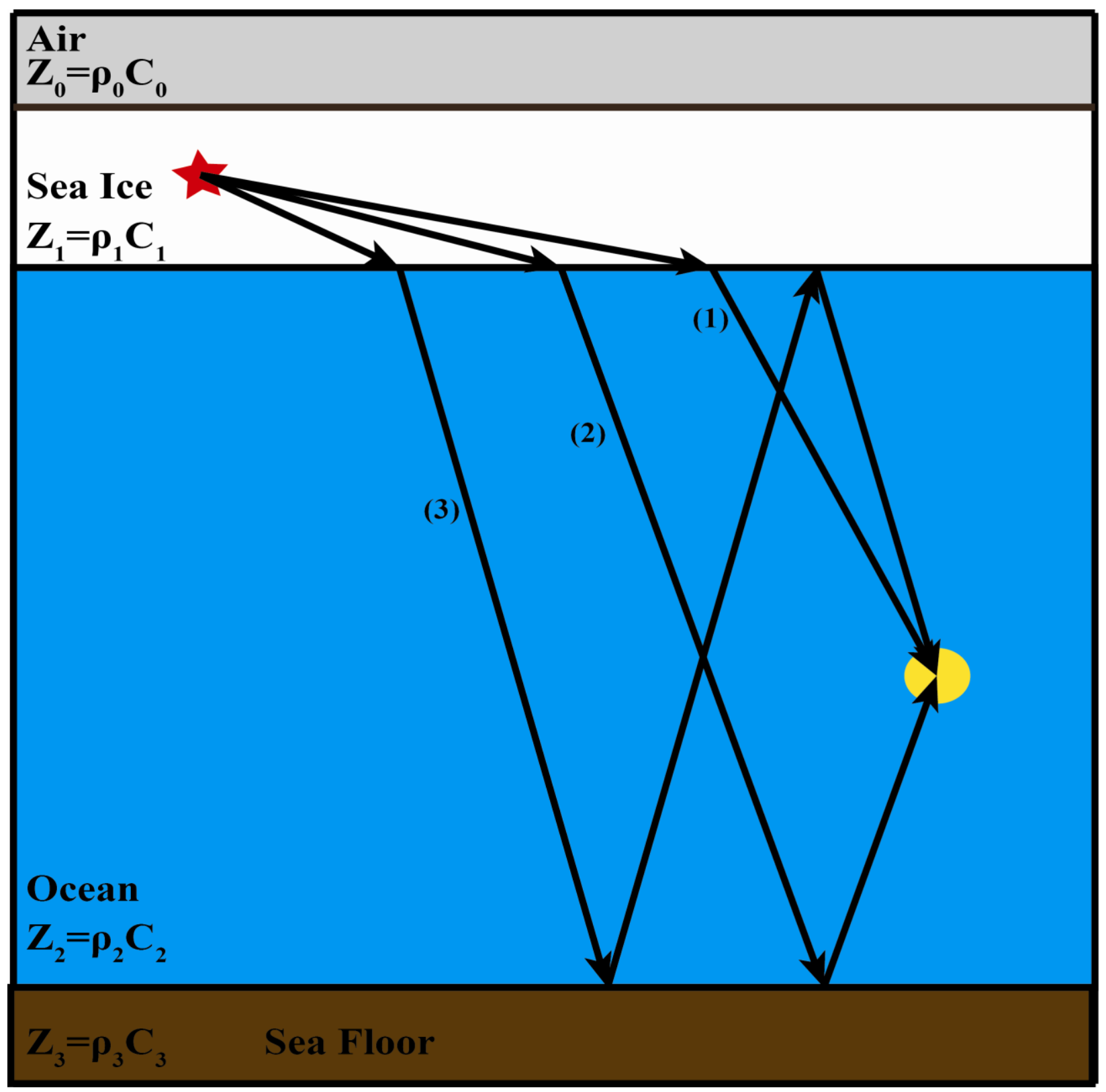

Figure 2.9-Waterborne acoustic paths between source (red star) and hydrophone (yellow circle). Ray (1) is the shortest path with one boundary interaction. Ray (2) is the second shortest path with two boundary interactions. Ray (3) is the longest path with three boundary interactions.

Waterborne paths are comprised of the lower frequencies that leak out of the waveguide along with any remaining frequencies that are transmitted, thus the waterborne paths can be compressed of all frequencies. 


\section{Chapter 3}

\section{OASN: NOISE, COVARIANCE MATRICES AND SIGNAL REPLICAS}

\subsection{Implementation}

To simulate the wavefront produced by a tensile crack in OASN: Noise, Covariance Matrices and Signal Replicas [10], discrete point sources were placed within 4 meter thick sea ice. The geometry for all simulations is shown in Figure 3.1.

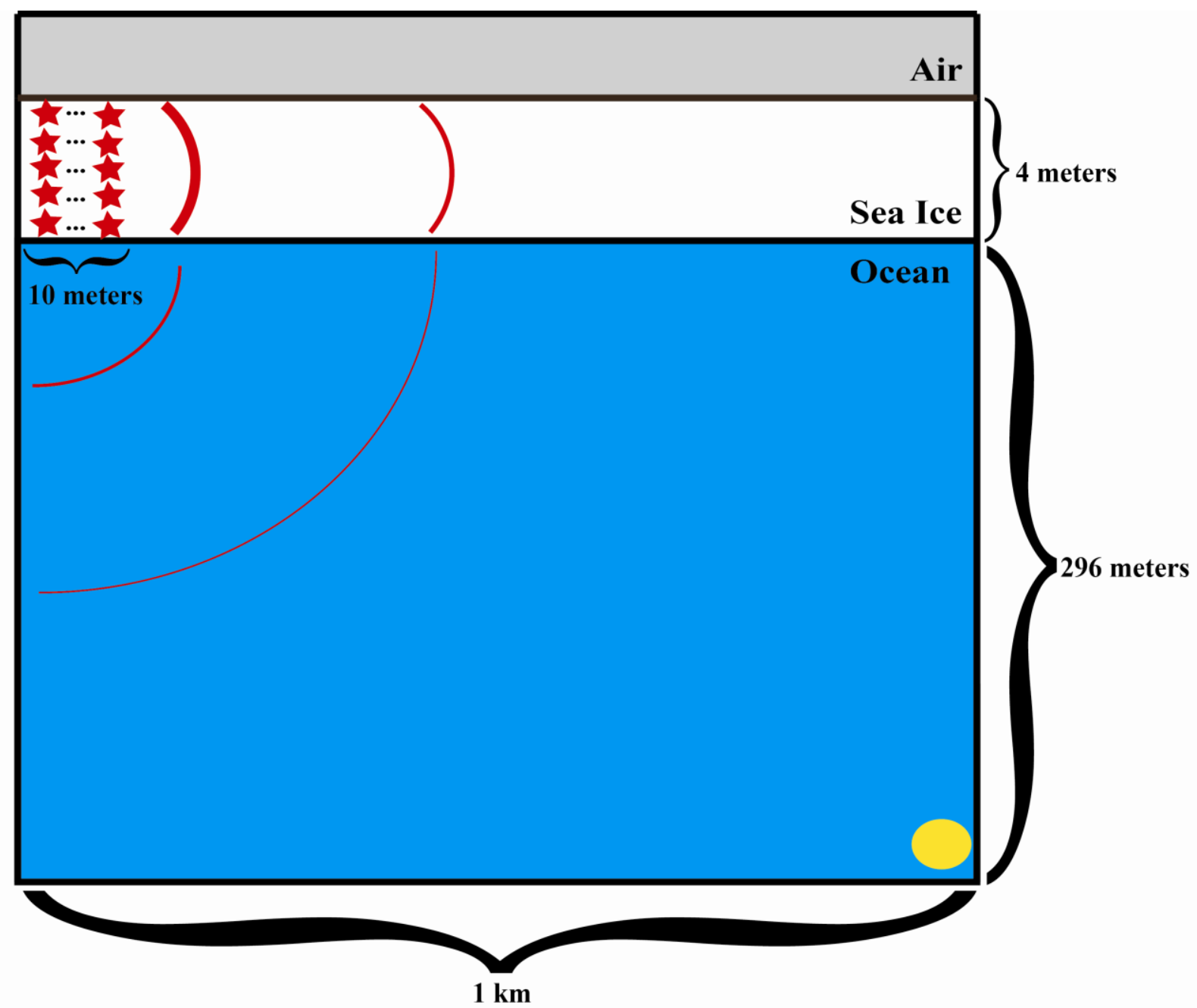

Figure 3.1 - Geometry for all simulations conducted in this study. Red stars represent discrete point sources whereas the yellow circle represents the hydrophone. The wavefront from the point sources are shown as red arcs. 
The sources were separated by a spatial Nyquist of 0.1 meters while the frequency range for simulations was computed over $2^{-52}$ to $5000 \mathrm{~Hz}$. A crack length of 10 meters was used. In order to efficiently simulate a variety of tensile crack propagations, a grid of points with a spatial Nyquist of 0.1 meters in the range and depth directions was simulated. For the 4 meter thick sea ice and a 10 meter long crack, 4,141 discrete sources were placed into a grid and simulated individually. The compressional sound speed for sea ice was 3500 m/s [8]. Propagation crack velocity $\left(\mathrm{V}_{\text {jump }}\right.$ ) ranged from $50 \mathrm{~m} / \mathrm{s}$ to $550 \mathrm{~m} / \mathrm{s}$. Jumps ranged from every 2 meters to 4 meters of crack propagation, whereas the pauses between jumps ranged from $0.1 \mathrm{~ms}$ to $2.0 \mathrm{~ms}$. The depth of the hydrophone was 300 meters for all simulations. The maximum range of a discrete source away from the hydrophone was 1 kilometer and a sea floor was not present. The range of 1 kilometer was chosen to decrease simulation time. By choosing a range of 1 kilometer the complete oscillatory habit of acoustics, due to the Munk profile Figure 2.6, propagating in the deep ocean did not need to be considered, The Munk sound speed profile was truncated to the hydrophone depth and thus a sea floor was not needed. The shear velocity of the sea ice was zeroed to simplify the model and eliminate the possibility of mode conversion. Any signal loss in the simulations was due to spreading loss which is inherently built into OASN. Attenuation was not considered within the model for simplification and due to the fact that OASN only allows attenuation to be entered as a function of distance away from the source, when in reality attenuation is a function of distance away from the source and frequency.

\subsection{Wavenumber Integration Induced Aberrations}

During the initial phases of this thesis, simulations from OASN had an aberration in the form of an acoustic arrival before the first expected arrival through the leaky waveguide. In this section 
the goal is to demonstrate with concise mathematics why OASN has an aberration. Figure 3.2 and Figure 3.3 demonstrate the aberration which was comprised of the full bandwidth of frequencies simulated at the exact time interval corresponding to the difference between the depth of the source and the depth of the hydrophone, with appropriate compressional sounds speeds between subsequent layers from the source to the hydrophone. It is not the goal to give a step-by-step derivation of the mathematics governing the aberration, however if such is required by the reader, they are referred to reference [16].

Starting from the nonhomogeneous Helmholtz equation in cylindrical coordinates for a range independent environment,

$$
\left[\nabla^{2}+k^{2}(z)\right] \psi(r)=f(r)
$$

, with the wavenumber $(\mathrm{k})$, the displacement of the propagating spherical wave $(\psi)$ and the source (f). To solve for the nonhomogeneous solution to (3.1) it first must be recognized that (3.1) is in the general from of equation,

$$
\boldsymbol{L u}(\boldsymbol{r})=\boldsymbol{f}(\boldsymbol{r})
$$




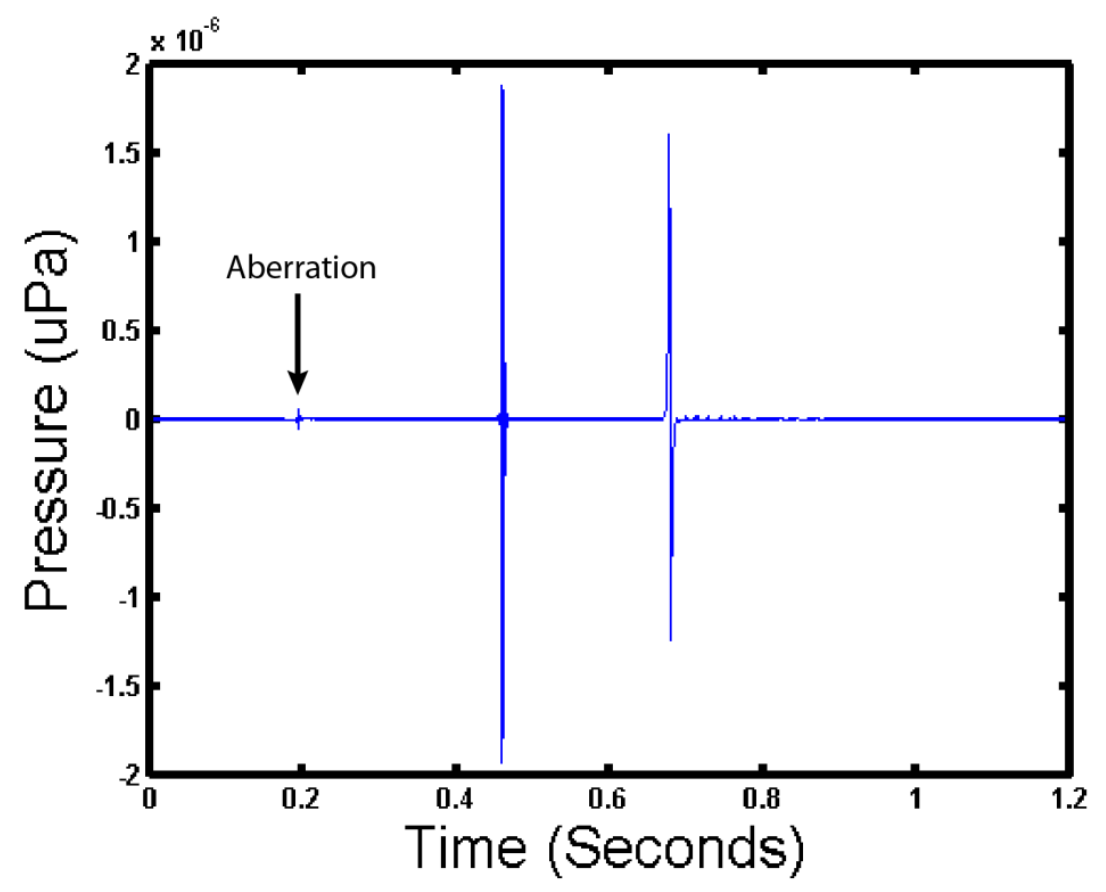

Figure 3.2 - Simulated time series with an aberration located around 0.2s.

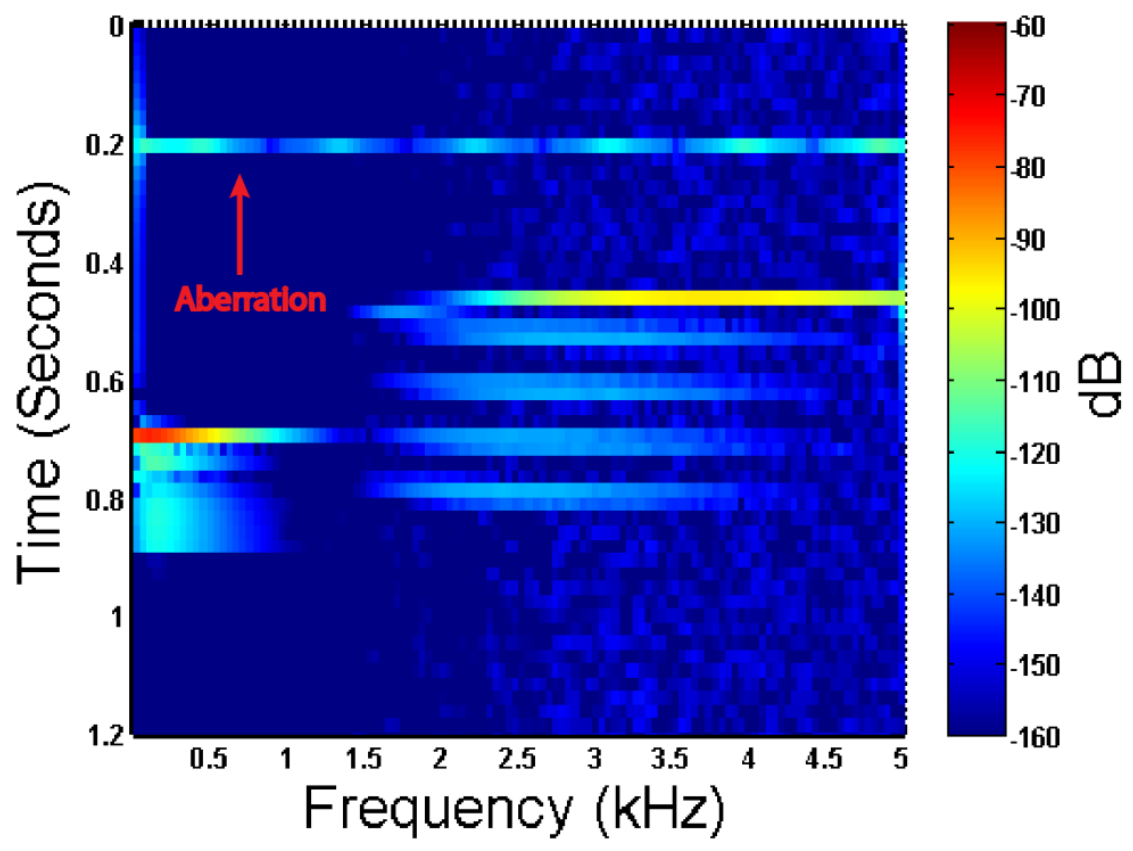

Figure 3.3 - Spectrum of the simulated time series in Figure 3.2 with an aberration located around 0.2 seconds comprised of the full bandwidth of frequency for the simulation. 
The nonhomogeneous solution to (3.1) can be solved with a Green's function as (3.1) is in the form (3.2). The solution to the nonhomogeneous Helmholtz equation,

$$
g\left(r, r_{0}\right)=A^{\prime}(r) \frac{e^{-i k(z) R}}{R} \text { with } R=\left|r-r_{0}\right|
$$

, is a Green's function of a spherical wave with $\mathrm{r}_{0}$ being the impulse at a radial coordinate. The modified specified amplitude, $\mathrm{A}^{\prime}(\mathrm{r})$, of (3.3) is an arbitrary constant that would satisfy the boundary condition between the source and the medium, while also containing any other constants, such as $\pi$ and 4, that appear readily in the mathematics. Since (3.3) is a Green's function, the solution to the nonhomogeneous Helmholtz equation can be written as,

$$
\left[\nabla^{2}+k^{2}(z)\right] g\left(r, r_{0}\right)=-\delta\left(r-r_{0}\right)
$$

,with the Dirac delta $(\delta)$ being the associated impulse response to (3.1).

The depth-separated wave equation,

$$
\left[\frac{d^{2}}{d z^{2}}+\left(k^{2}-k_{r}^{2}\right)\right] \psi\left(k_{r}, z\right)=-\delta\left(z-z_{s}\right)
$$

, is a rearrangement of the nonhomogeneous Helmholtz equation via a variable integration with respect to the azimuthal coordinate $(\varphi)$ and an application of the forward and reverse zeroethorder Hankel transforms,

$$
\begin{aligned}
& F\left(k_{r}, z\right)=\int_{0}^{\infty} f(r, z) J_{0}\left(k_{r} r\right) r d r \quad \& \\
& f(r, z)=\int_{0}^{\infty} F\left(k_{r}, z\right) J_{0}\left(k_{r} r\right) k_{r} d k_{r}
\end{aligned}
$$

, allowing for the radial or range dependency to be dropped as a variable in favor of depth (z) dependency along with wavenumber $(\mathrm{k})$, radial wavenumber $\left(\mathrm{k}_{\mathrm{r}}\right)$ and source impulse $\left(\mathrm{z}_{\mathrm{s}}\right)$. The radial wavenumber is defined as, $\mathrm{k}_{\mathrm{r}} \equiv \mathrm{k} \cos (\theta)$. Equation (3.5) can be said to be in the k-domain 
or wavenumber domain, as the radial dependency is technically still there but has been transformed into wavenumber space via a Hankel transform. To solve the depth-separated wave equation, a boundary value problem approach must be taken, thus the homogenous solution to the depth-separated wave equation must be found. The homogenous solution to (3.5) should be in the form of, (2.1), a spherical wave. The homogenous solution,

$$
H\left(k_{r}, z\right)=A^{I^{+}}\left(k_{r}\right) e^{i z \sqrt{k^{2}-k_{r}^{2}}}+A^{I^{-}}\left(k_{r}\right) e^{-i z \sqrt{k^{2}-k_{r}^{2}}}
$$

, of the depth-separated wave equation can be found by directly solving the Helmholtz equation using a characteristic equation, obtaining complex roots with a real number of zero and eliminating the sine function, as it is zero at the origin, and finally rewriting the remaining cosine function in terms of its complex identity. Notice that the square root expression in (3.8) could have been written in terms of the wavenumber $\mathrm{k}_{\mathrm{z}}$ as by definition $\mathrm{k}_{\mathrm{z}} \equiv \sqrt{k^{2}-k_{r}^{2}}$. The depthseparated wave equation can be solved by a Green's function as it is in the form of,

$$
L G(x, s)=-\delta(x-s)
$$

, thus implying that a Green's function exists. The particular solution is,

$$
\mathbf{G}\left(\mathbf{k}_{\mathrm{r}}, \mathbf{z}, \mathbf{z}_{\mathrm{s}}\right)=\mathbf{A}^{\prime}\left(\mathbf{k}_{\mathrm{r}}\right) \mathrm{e}^{\mathrm{i}\left|\mathrm{z}-\mathrm{z}_{\mathrm{s}}\right| \sqrt{\mathbf{k}^{2}-\mathbf{k}_{\mathbf{r}}^{2}}}
$$

, with $A^{\prime}\left(k_{r}\right)$ being the arbitrary constant used to satiate the boundary conditions and remaining constants and $\mathrm{z}_{\mathrm{s}}$ being the impulse at the depth coordinate. The complete solution,

$$
\Psi\left(\mathbf{k}_{\mathbf{r}}, \mathbf{z}, \mathbf{z}_{\mathrm{s}}\right)=\mathbf{H}\left(\mathbf{k}_{\mathbf{r}}, \mathbf{z}\right)+\mathbf{G}\left(\mathbf{k}_{\mathrm{r}}, \mathbf{z}, \mathbf{z}_{\mathrm{s}}\right)
$$

, and Green's function, to the depth-separated wave equation, (3.5), is the addition of the homogenous solution and the particular solution. Finally, applying (3.7) to (3.10) yields 


$$
\mathbf{g}\left(\mathbf{r}, \mathbf{z}, \mathbf{z}_{\mathrm{s}}\right)=\int_{0}^{\infty} A^{\prime}\left(\mathbf{k}_{\mathbf{r}}\right) \mathbf{e}^{\mathrm{i}\left|\mathbf{z}-\mathbf{z}_{\mathrm{s}}\right| \sqrt{\mathbf{k}^{2}-\mathbf{k}_{\mathbf{r}}^{2}}} J_{0}\left(\mathbf{k}_{\mathbf{r}} \mathbf{r}\right) \mathbf{k}_{\mathbf{r}} \mathbf{d} \mathbf{k}_{\mathbf{r}}
$$

For the simulations run in this study, OASN evaluates the integral of (3.12) via a numerical method called wavenumber integration [16]. The vital component to understanding how the aberration manifests itself is to understand the radial wavenumber and its influences on the zeroth-order $\mathrm{J}^{\text {th }}$ Bessel function. OASN requires the input of a minimum and maximum phase speed used to steer the direction of the impulse received at the response in wavenumber space, 360 degrees, effectively allowing for a point source to be placed at a specified depth but arbitrary radial component dictated by the radial wavenumber $\mathrm{k}_{\mathrm{r}}$. The specified phase speeds do not, however, correlate to the phase velocities of the spherically propagating wave directly. In wavenumber space $\mathrm{k}_{\mathrm{r}}=\mathrm{k} \cos (\theta)$ and $\mathrm{k}_{\mathrm{z}}=\mathrm{k} \sin (\theta)$. The direct path from immediately above the hydrophone corresponds to an angle near perpendicular to the horizontally stratified layers, around 90 degrees when the impulse is above the response spatially. However, OASN also uses the wavenumber space to truncate and sample the definite integral of (3.12) from zero to infinity. For example, if a minimum phase speed of $1450 \mathrm{~m} / \mathrm{s}$ is entered with a maximum phase speed of $1 \times 10^{9} \mathrm{~m} / \mathrm{s}$, the calculation of steering angles 0 degrees and 90 degrees would correspond to

$$
\begin{aligned}
& \cos ^{-1}\left(\frac{1450 \mathrm{~m} / \mathrm{s}}{1450 \mathrm{~m} / \mathrm{s}}\right)=0 \text { radians \& } \\
& \cos ^{-1}\left(\frac{1450 \mathrm{~m} / \mathrm{s}}{1 \times 10^{9} \mathrm{~m} / \mathrm{s}}\right) \approx \frac{\pi}{2}
\end{aligned}
$$

, respectively. As OASN samples between the minimum and maximum wavenumbers in wavenumber space it also samples between a truncated range between zero and infinity. Accordingly, the maximum phase speed or minimum wavenumber in wavenumber space corresponds to zero in the wavenumber integral of equation (3.12) whereas and the minimum 
phase speed or maximum wavenumber in wavenumber space corresponding to infinity in the wavenumber integral. When OASN is sampling near the maximum phase speed, the steering angle is near perpendicular to the horizontally stratified layers and the wavenumber in wavenumber space is at a minimum. As a result, the truncated interval between 0 and infinity is being sampled at zero. With the wavenumber being evaluated at zero in wavenumber space, the zeroth-order $\mathrm{J}^{\text {th }}$ Bessel function is at the origin. The zeroth-order $\mathrm{J}^{\text {th }}$ Bessel function is bounded at the origin and oscillatory going toward a value of unity as it approaches the origin. As values near $\mathrm{J}_{0}(0)$ are calculated and integrated within the wavenumber integral, the values toward the origin of the zeroth-order $\mathrm{J}^{\text {th }}$ Bessel function have a larger influence than those proceeding them. If the wavenumber integral in not zeroed and the discontinuity is not smoothed outside of an interval where the zeroth-order

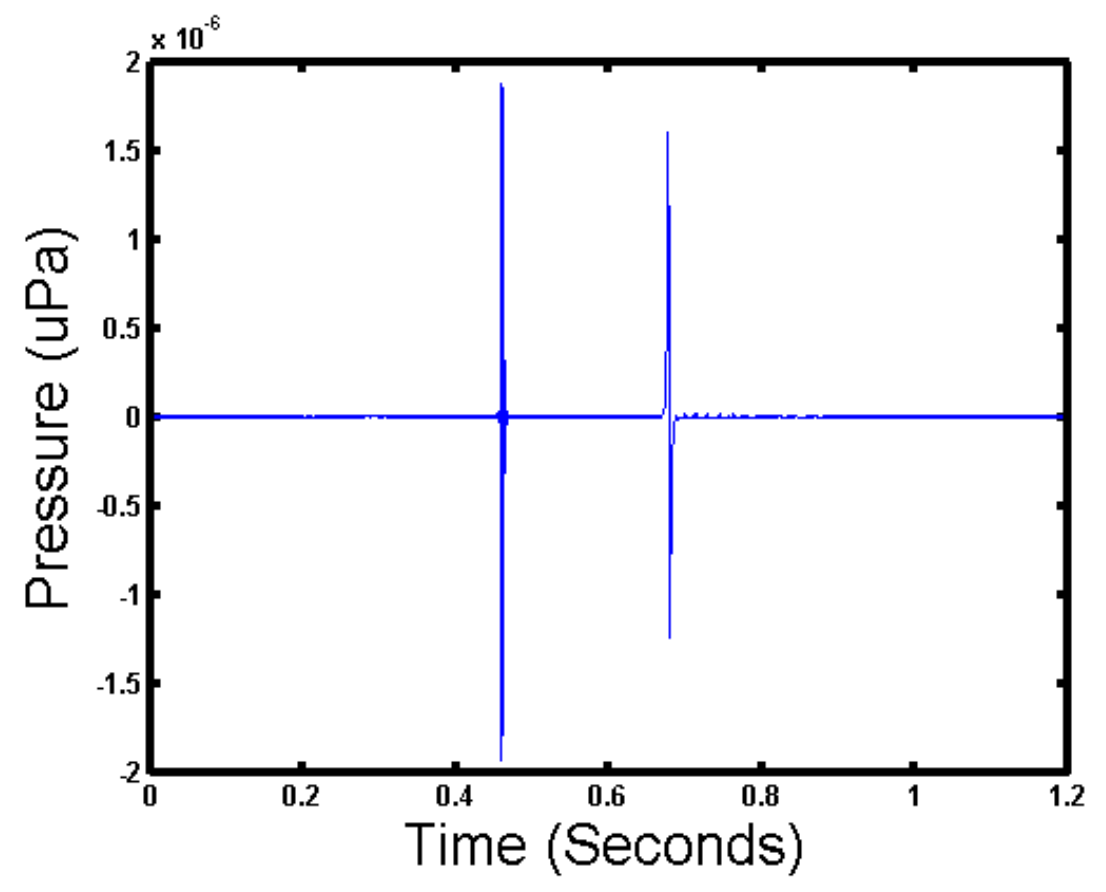

Figure 3.4 - Simulated time series with the aberration removed. The geometry of the simulation is identical to that in Figure 3.2. 


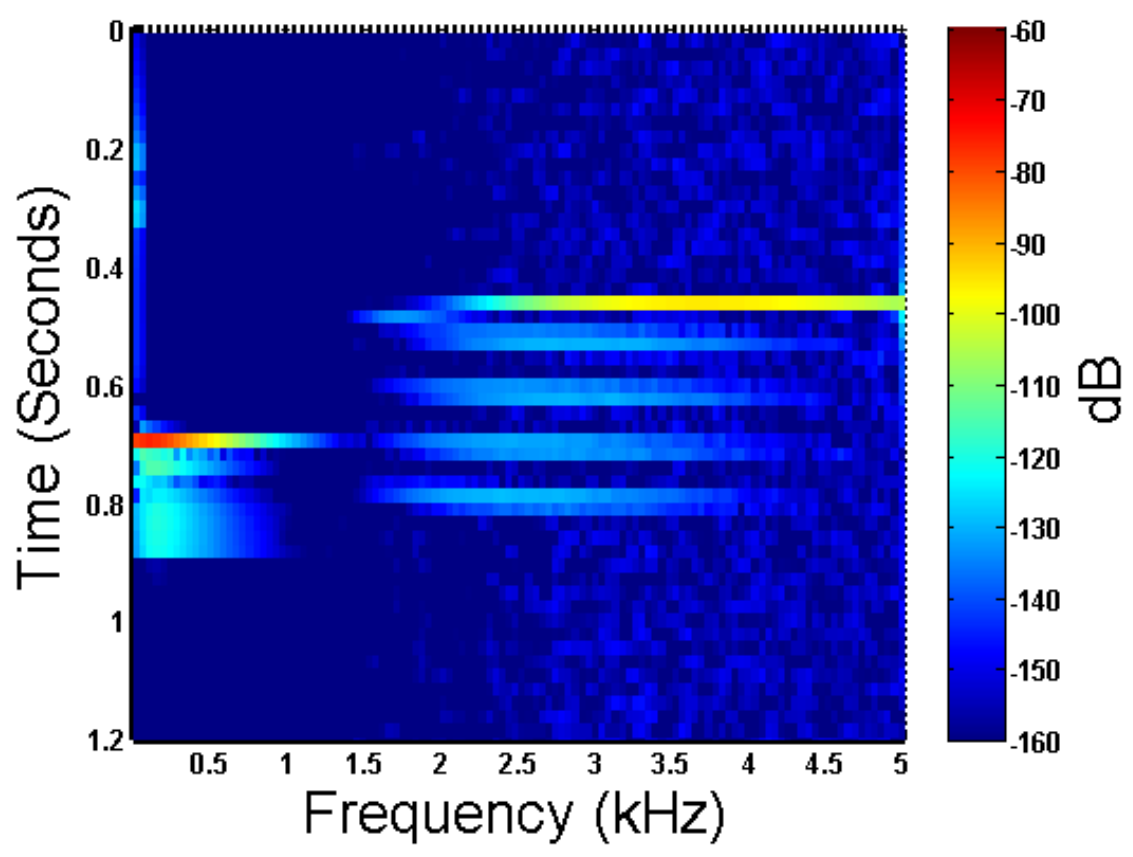

Figure 3.5 - Spectrogram of the simulated time series in Figure 3.4. The geometry of the simulation is identical to that of Figure 3.2.

$\mathbf{J}^{\text {th }}$ Bessel function has values that are similar in magnitude, aberrations will appear at the steering angle corresponding to such wavenumber in wavenumber space. To reduce the aberration, the sampling between the minimum and maximum phase speeds is truncated so that sampling toward the minimum phase speed is minimized, effectively bring the minimum phase speed closer to that of the maximum phase speed. The reduced aberration is shown in Figure 3.4 and Figure 3.5.

Zeroing and smoothing the discontinuity between an interval in wavenumber space for the zeroth-order $\mathbf{J}^{\text {th }}$ Bessel function can, however, cause a secondary issue. By truncating the sampling space between the minimum and maximum phase speed intervals, the minimum phase speed is brought closer to the maximum phase speed. As the difference between the minimum and maximum phase speeds becomes smaller, the evaluation of (3.14) moves away from 90 
degrees and toward zero degrees as the ratio between the numerator and dominator or minimum and maximum phase speed approaches unity. As this happens, the waterborne propagating waves become weighted more over those of the leaky waveguide.

\subsection{OASN Comparison with RAM}

To validate the reduction of the aberration and the output of OASN, a comparison was made with simulations computed using the Range-dependent Acoustic Model (RAM) [11]. The geometry for the simulations was a range of 300 meters, a depth of 112 meters, sea ice thickness of 16 meters, and a single source placed 2 meters below the sea ice. The hydrophone was 200 meters in range away from the source. Identical geometry with frequencies ranging from $25 \mathrm{~Hz}$ to $4000 \mathrm{~Hz}$ was run in both OASN and RAM. The goal of this exercise was to see if OASN was producing appropriate arrival times of the propagating spherical waves along with validating the

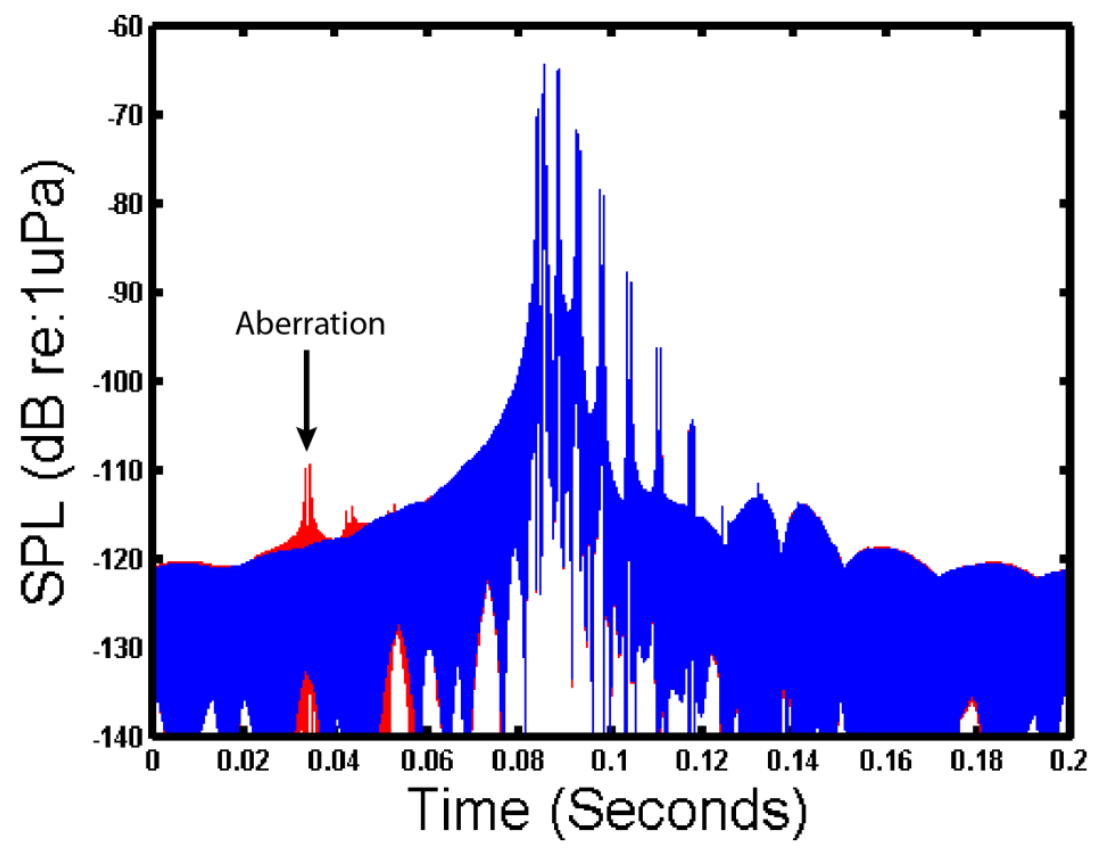

Figure 3.6 - Simulated Sound Pressure Level (SPL) comparison of identical geometry between OASN with aberration (Red) and OASN without aberration (blue). 


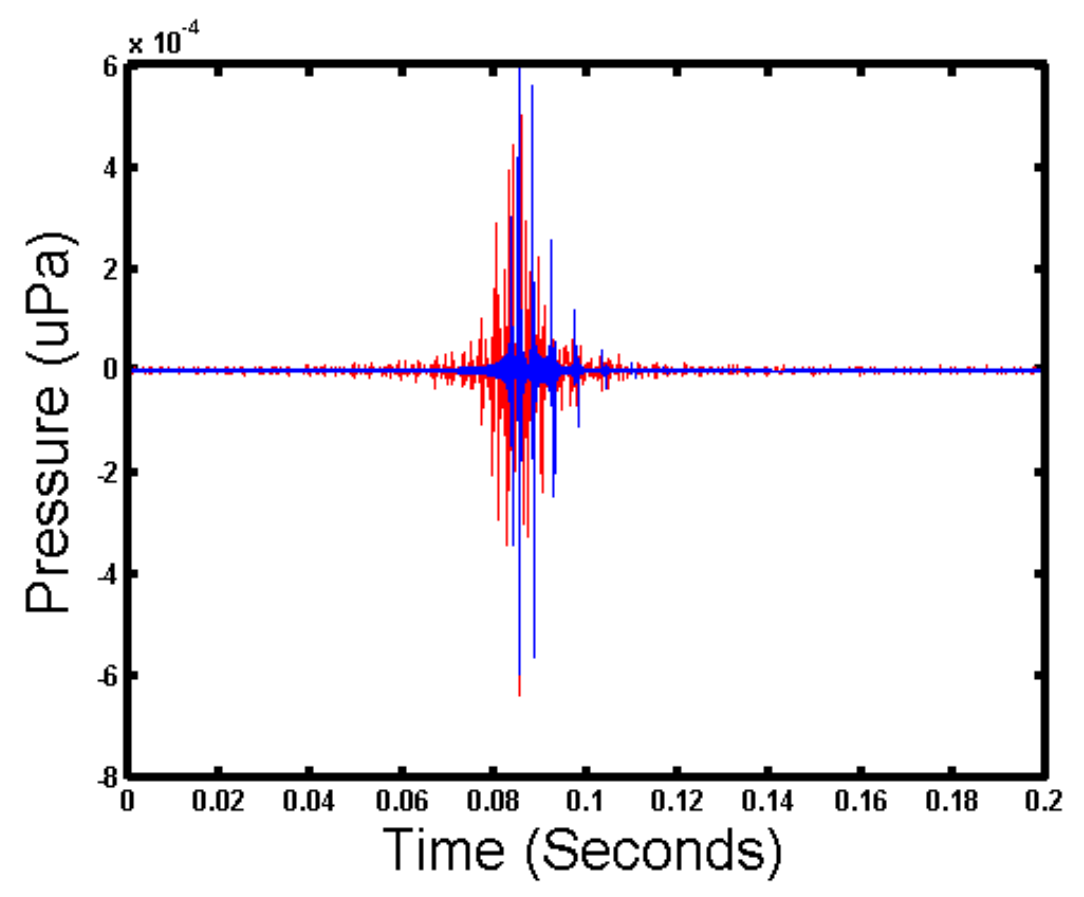

Figure 3.7 - Simulated time series comparison between OASN with aberration reduced (blue) and RAM (red) for a single source within sea ice.

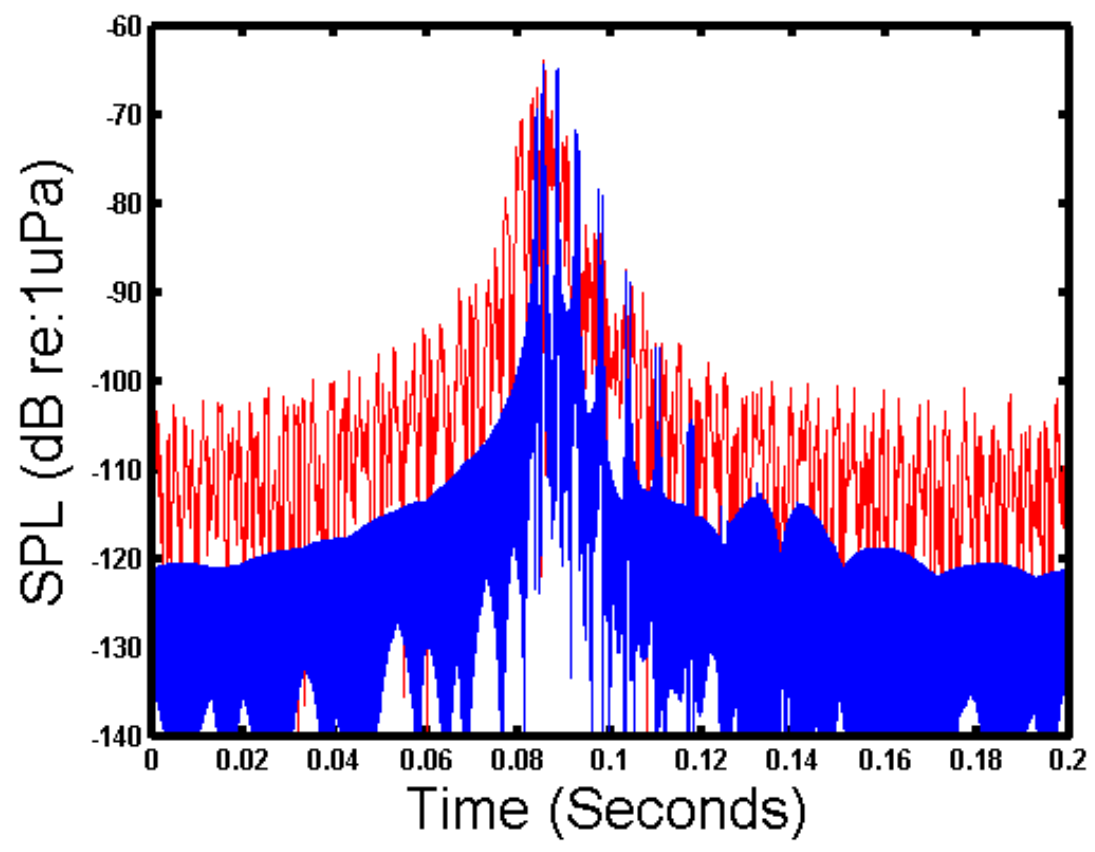

Figure 3.8 - Simulated Sound Pressure Level (SPL) comparison between OASN with aberration reduced (Blue) and RAM (red). The geometry of the simulation is identical to that of Figure 3.7. 
methods used to reduce the aberration. RAM was chosen as the software for comparison for two reasons: first, RAM solves a forward propagating wave equation as opposed to the depthseparated wave equation solved with OASN, and second, RAM was extensively used by one of the NEAR-Lab personnel at Portland State University for a project involving point source discretization to simulation the acoustic waves generated from driving piles into river sediments. Therefore, the previous extensive use of RAM allowed for a greater degree of confidence to be placed on its outputs, as opposed to OASN which demonstrates a clear aberration when attempting to model the geometry presented within this study. By comparing the outputs of two similar software packages that use two different approaches for solving the wave equation, this study explores the idea that if a correlation in the data between OASN and RAM could be drawn, then the reduction of the aberration and the implementation of OASN for modeling tensile cracks within sea ice would be validated. Figure 3.6 is a Sound Pressure Level comparison between OASN with and without the aberration reduced. Figure 3.7 and Figure 3.8 are a comparison of the simulated time series and Sound Pressure Level (SPL) from OASN, with the aberration reduced, and RAM, respectively. In the comparison, the arrival times for the primary arrival of the propagating spherical wave was almost identical between OASN and RAM. Thus, the reduction of the aberration can be considered successful as the presence of the aberration had been reduced whereas the arrival times and SPL of the spherical waves were similar between OASN and RAM. 


\section{Chapter 4}

\section{Simulation Data and Comparison to Observed Ambient Noise}

\subsection{Simulation Data}

To cover a range of tensile crack propagations, four simulations are presented to demonstrate the variance in simulated time series and, therefore, spectrograms from similarly propagating tensile cracks. For all simulations, the cracks are propagating toward the hydrophone. In all time series, multiple arrivals occur. The first peak or arrival is that of the pathgroupings corresponding to propagation through the leaky waveguide. The second peak is the waterborne path-groupings that propagate briefly through the sea ice, leak out of the waveguide and propagate primarily through the water.

Two tensile cracks are shown in Figures 4.1 through Figure 4.4. Each fracture penetrated completely though the 4 meter thick sea ice and had a fracture length of 10 meters. Jump distance was 2 meters and the fracture pause was $0.3 \mathrm{~ms}$. The simulations were identical, with the exception of the jump velocity $\left(\mathrm{V}_{\text {jump }}\right)$. The jump velocity was $550 \mathrm{~m} / \mathrm{s}$ for Figure 4.1 and Figure 4.2, whereas the jump velocity was $400 \mathrm{~m} / \mathrm{s}$ for Figure 4.3 and Figure 4.4. The differences between the cracks are more apparent in the time series, Figure 4.1 and Figure 4.3. Fractures with the higher velocity had a smaller pressure magnitude for leaky waveguide arrivals, but larger pressure magnitude of waterborne arrivals, when compared to an identical fracture with slower velocity. 


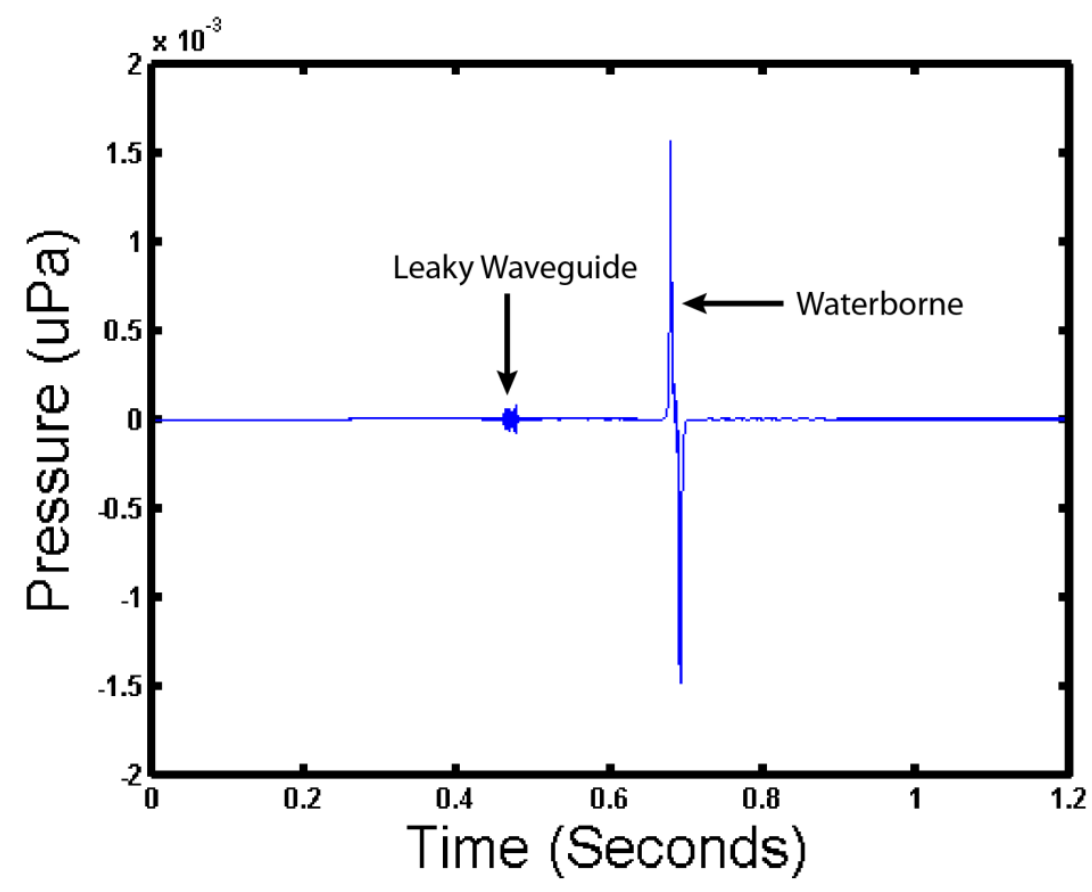

Figure 4.1 - Simulated time series of a tensile crack propagating toward the hydrophone at $550 \mathrm{~m} / \mathrm{s}$.

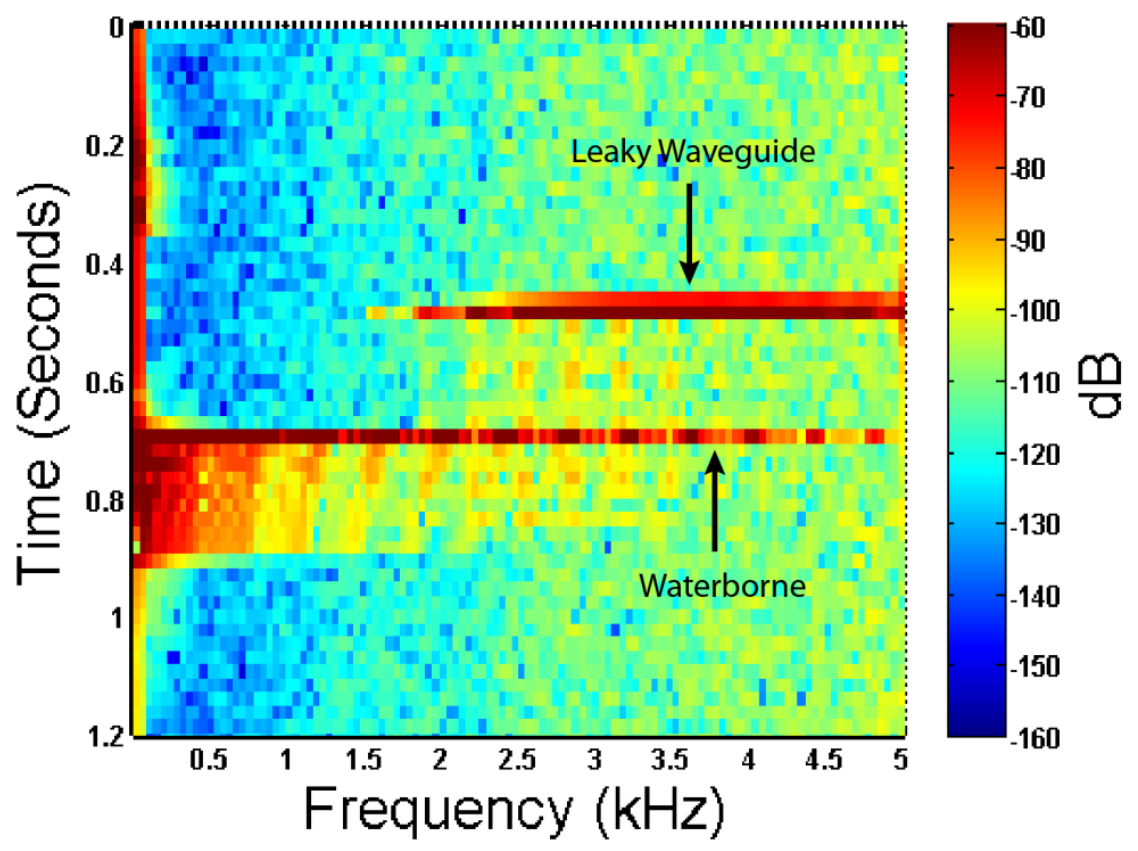

Figure 4.2 - Spectrogram of the simulated time series shown in Figure 4.1. 


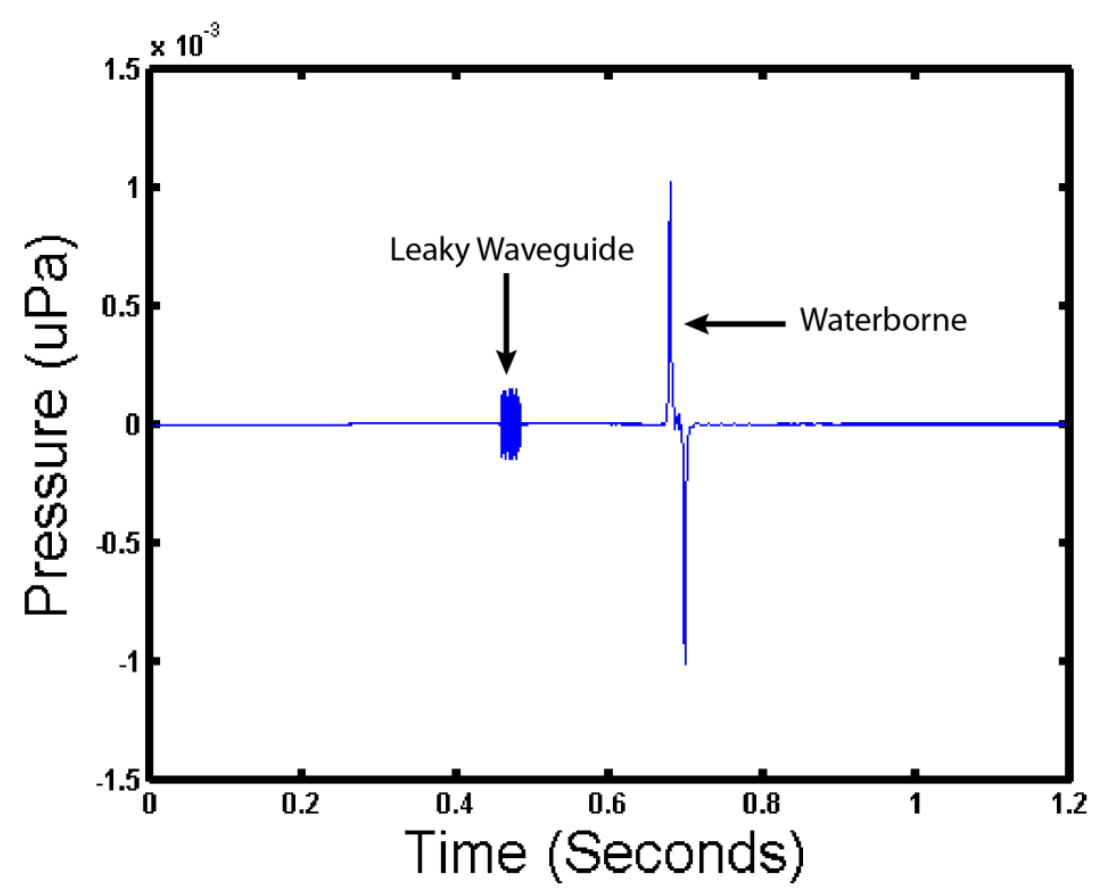

Figure 4.3 - Simulated time series of a tensile crack propagating toward the hydrophone at $400 \mathrm{~m} / \mathrm{s}$.

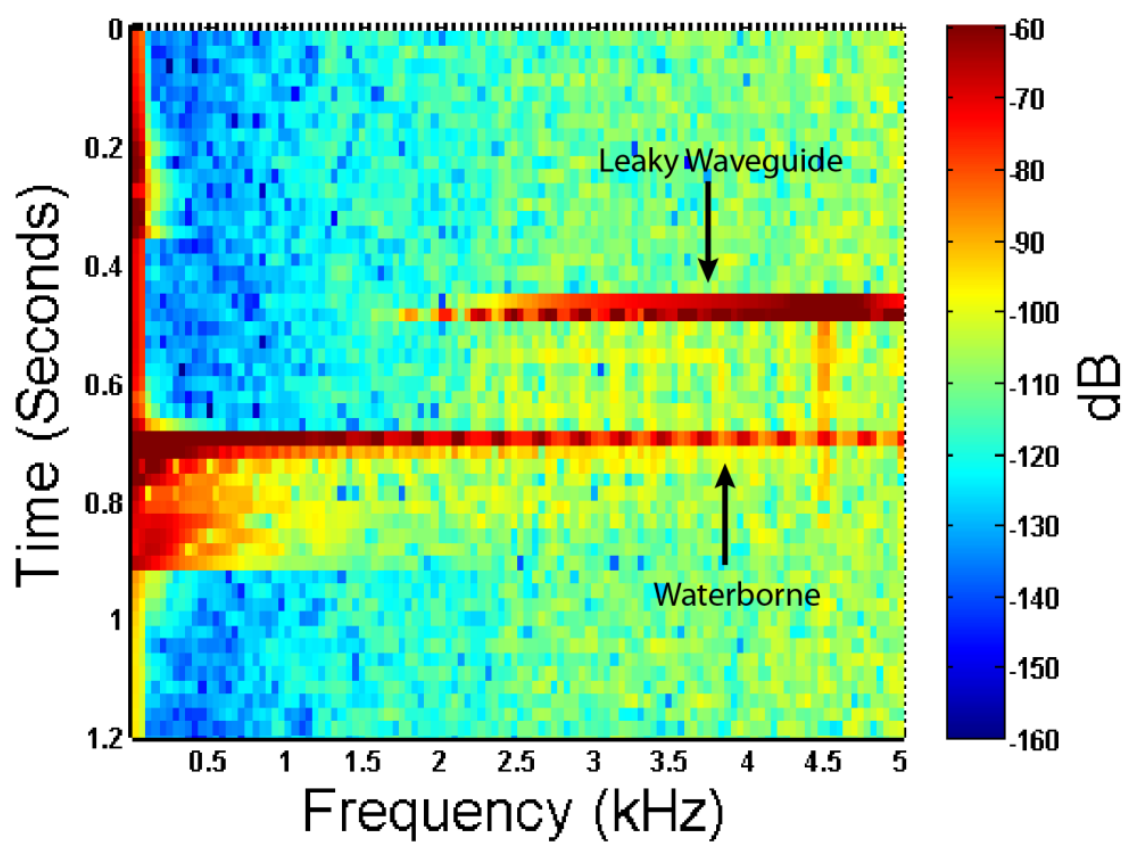

Figure 4.4 - Spectrogram of the simulated time series shown in Figure 4.3. 


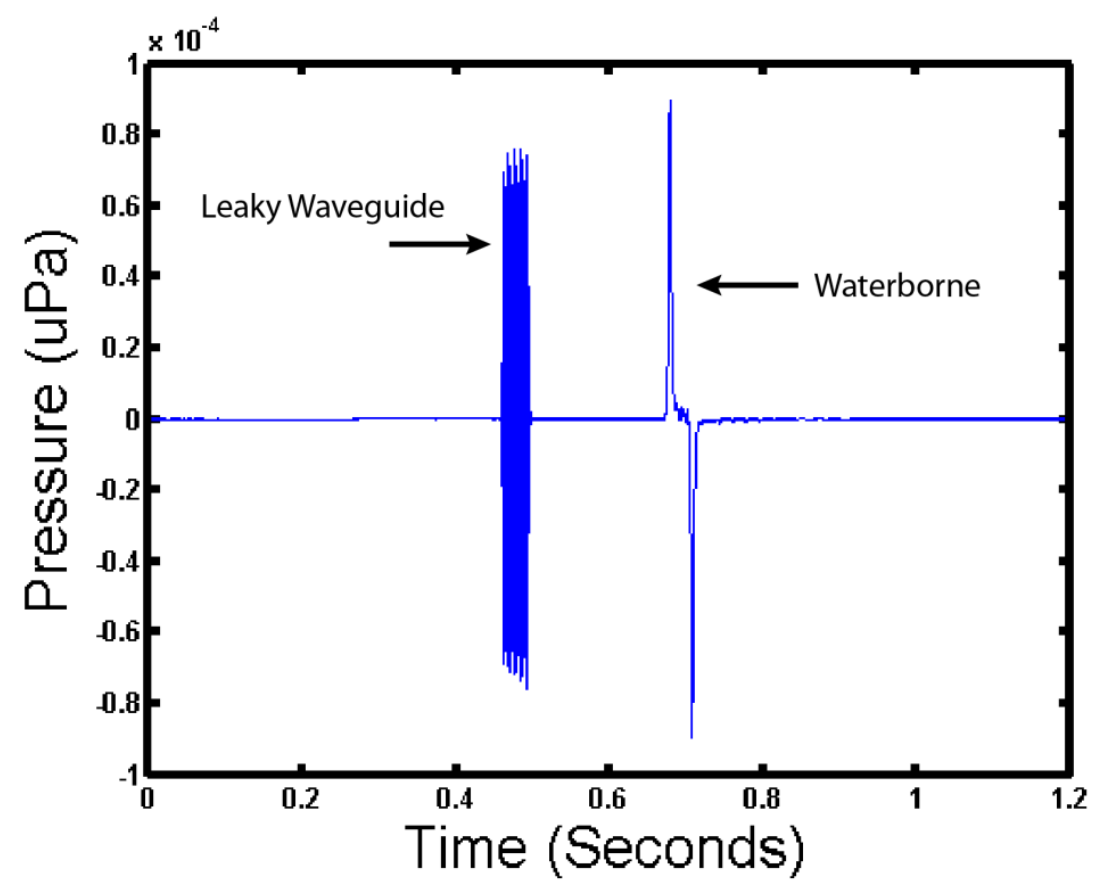

Figure 4.5 - Simulated time series of a shallow tensile crack propagating toward the hydrophone at $285 \mathrm{~m} / \mathrm{s}$.

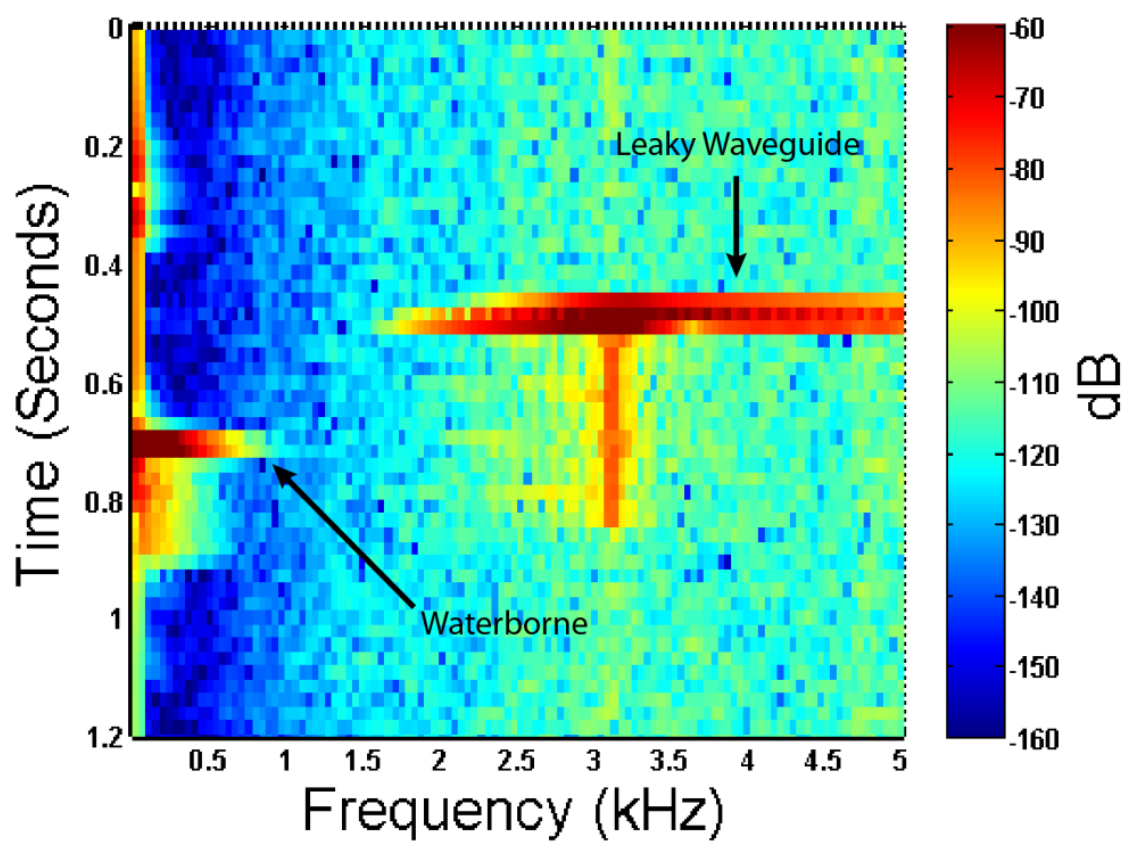

Figure 4.6 - Spectrogram of the simulated time series shown in Figure 4.5. 


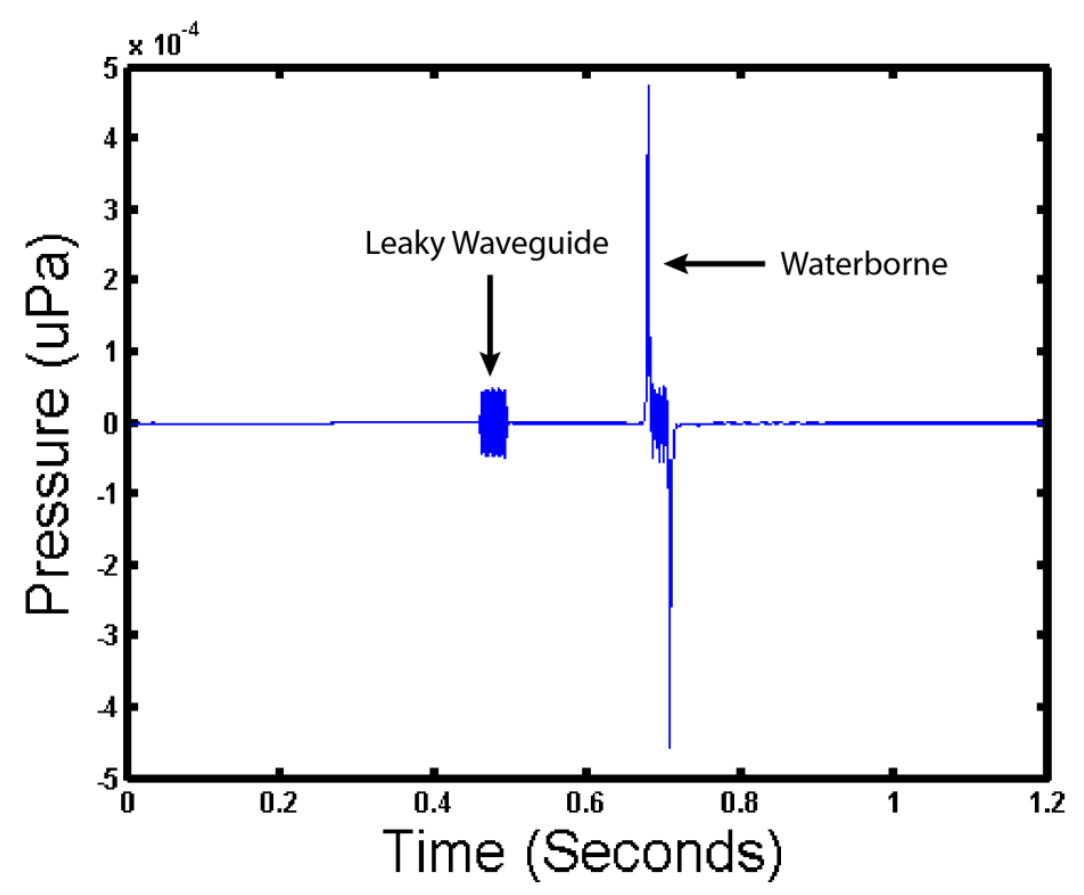

Figure 4.7 - Simulated time series of a deep tensile crack propagating toward the hydrophone at $285 \mathrm{~m} / \mathrm{s}$.

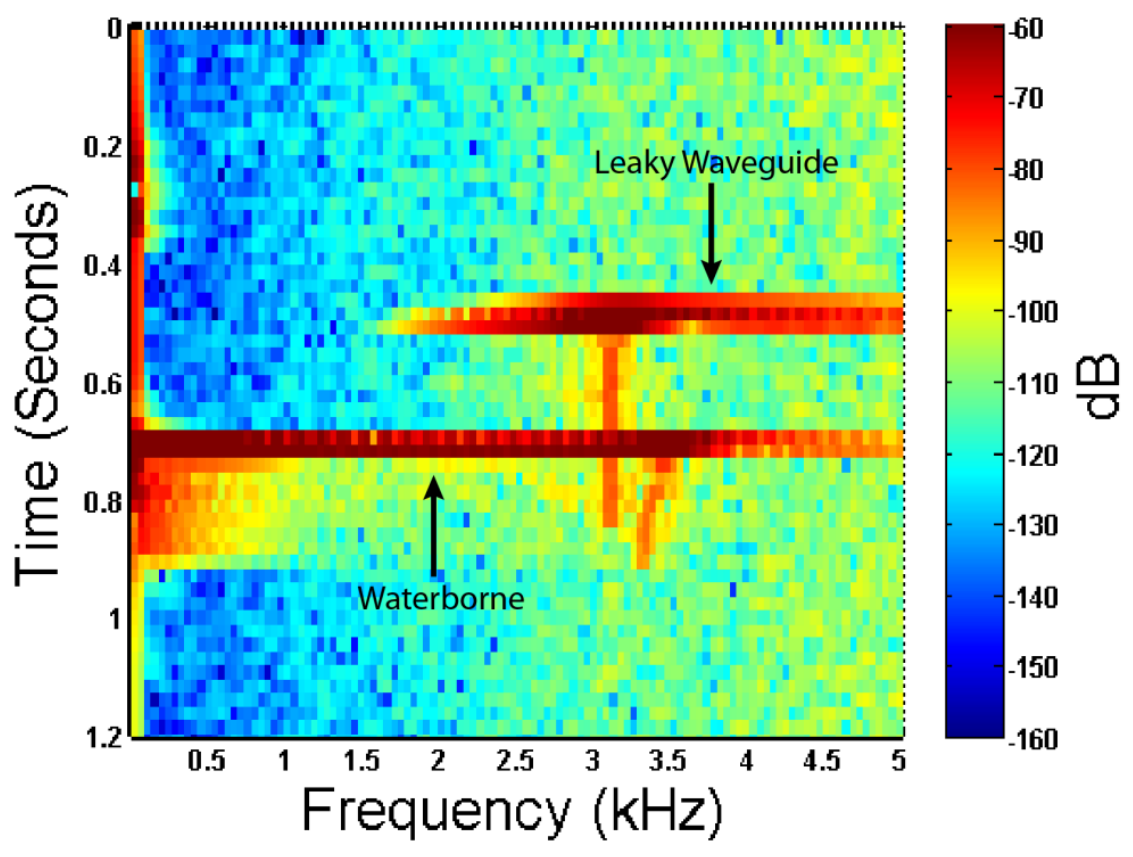

Figure 4.8 - Spectrogram of the simulated time series shown in Figure 4.7. 
The spectrograms of the higher velocity and slower velocity fractures, Figure 4.2 and Figure 4.4, showed similar behavior. Leaky waveguide arrivals were primarily of higher a frequency sound, while the waterborne arrivals had significant energy across the entire simulated frequency range. The cutoff frequency for the leaky waveguide is $437.5 \mathrm{~Hz}$. For both fractures, the lowest frequency for leaky waveguide arrivals is well above the cutoff frequency. However, the distribution of frequencies for the waveguide and waterborne arrivals was in agreement with waveguide characteristics.

Simulated shallow and deep tensile cracks are compared in Figures 4.5 through Figure 4.8. Jump distance was 2.5 meters and the fracture pauses was $0.4 \mathrm{~ms}$. Fracture lengths were 10 meters with fracture velocity $\left(\mathrm{V}_{\text {jump }}\right)$ at $285 \mathrm{~m} / \mathrm{s}$. Each fracture was at a depth of 1.7 meters into the ice. The difference between the two tensile cracks was the location of depth within the sea ice. The shallow fracture, Figure 4.5 and Figure 4.6, was within the upper 1.7 meters of the 4 meter thick sea ice -1.7 meters down from the air sea ice interface - whereas the deep fracture, Figure 4.7 and Figure 4.8, was in the bottom 1.7 meters of the sea ice -1.7 meters up from the sea ice water interface. The time series are compared in Figure 4.5 and Figure 4.7. The overall pressure magnitude of the leaky waveguide arrivals was the same between the shallow and deep paths. The waterborne arrivals, however, differred in pressure magnitude between the shallow and the deep fractures. The deep fracture had larger pressure for waterborne arrivals than the shallow fracture. Examaning the respective spectrograms for the shallow and deep fractures, Figure 4.6 and Figure 4.8, the leaky waveguide arrivals had similar frequency bandwidths situated in the higher frequencies. The leaky waveguide arrivals, comprised of the higher frequencies, were in agreement with waveguide characteristics. However, the lowest frequency of both leaky waveguide arrivals was well above the cutoff frequency of $437.5 \mathrm{~Hz}$. There was 
variance between the waterborne arrivals of the two fractures as the shallow fracture had a much narrower and lower frequency bandwidth, whereas deep fracture spanned the entire frequency range of the simulations.

\subsection{Comparison to Observed Ambient Noise}

The data used for comparison is uncalibrated single hydrophone data of sea ice deformation events that led to the emission of ambient noise into the water. The two events selected for comparison where chosen for their easily heard and visually spotted deformation events within the audio files, time series and spectrograms. The actual method of deformation, geometry and type of fracture are unknown. The significance of this comparison is to draw a correlation between the simulations and the observed deformation events present within sea ice.

The first deformation event, Figure 4.9 and Figure 4.10, is broadband in frequency. The time series, Figure 4.9, displays a single event similar to broadband waterborne arrivals for fractures that penetrated completely through the sea ice and fractures that were deep within the sea ice. The spectrogram, Figure 4.10, shows a range of frequencies comparable to the simulated frequencies range in this study for waterborne arrivals of tensile cracks that penetrated completely through the sea ice and fractures that were deep within the sea ice. 


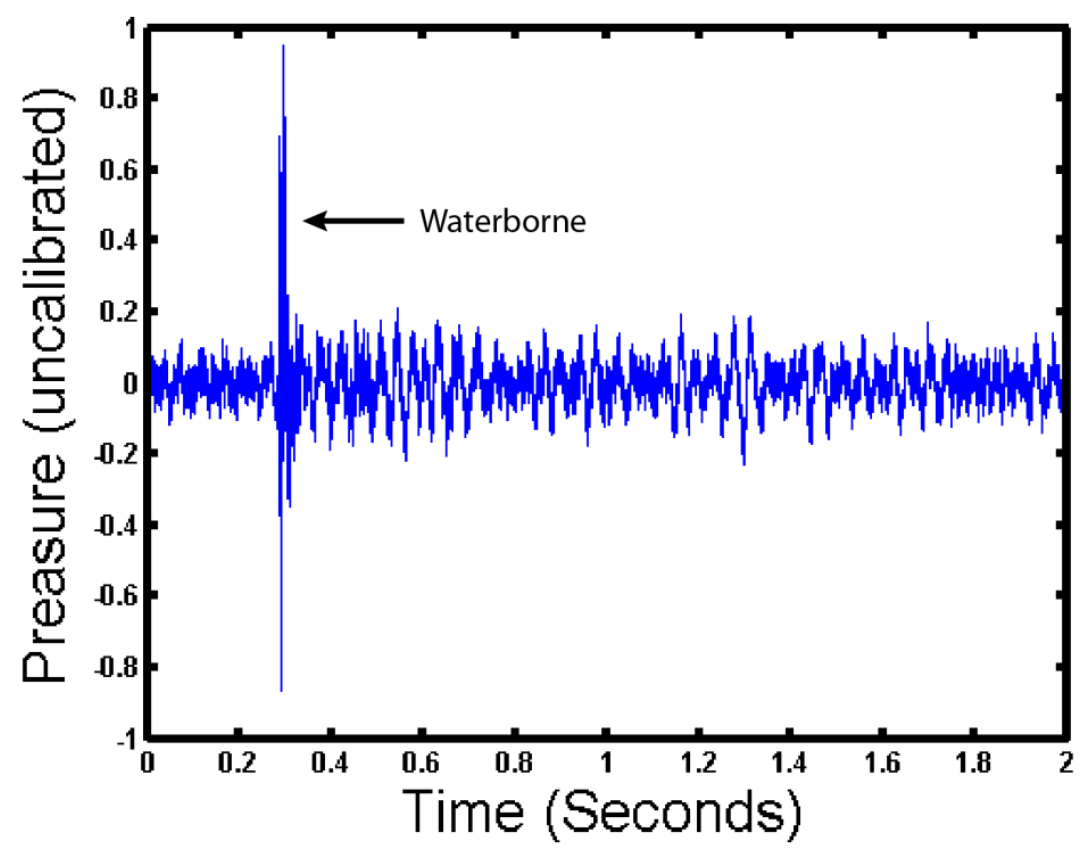

Figure 4.9 - Observed time series of sea ice deformation event one.

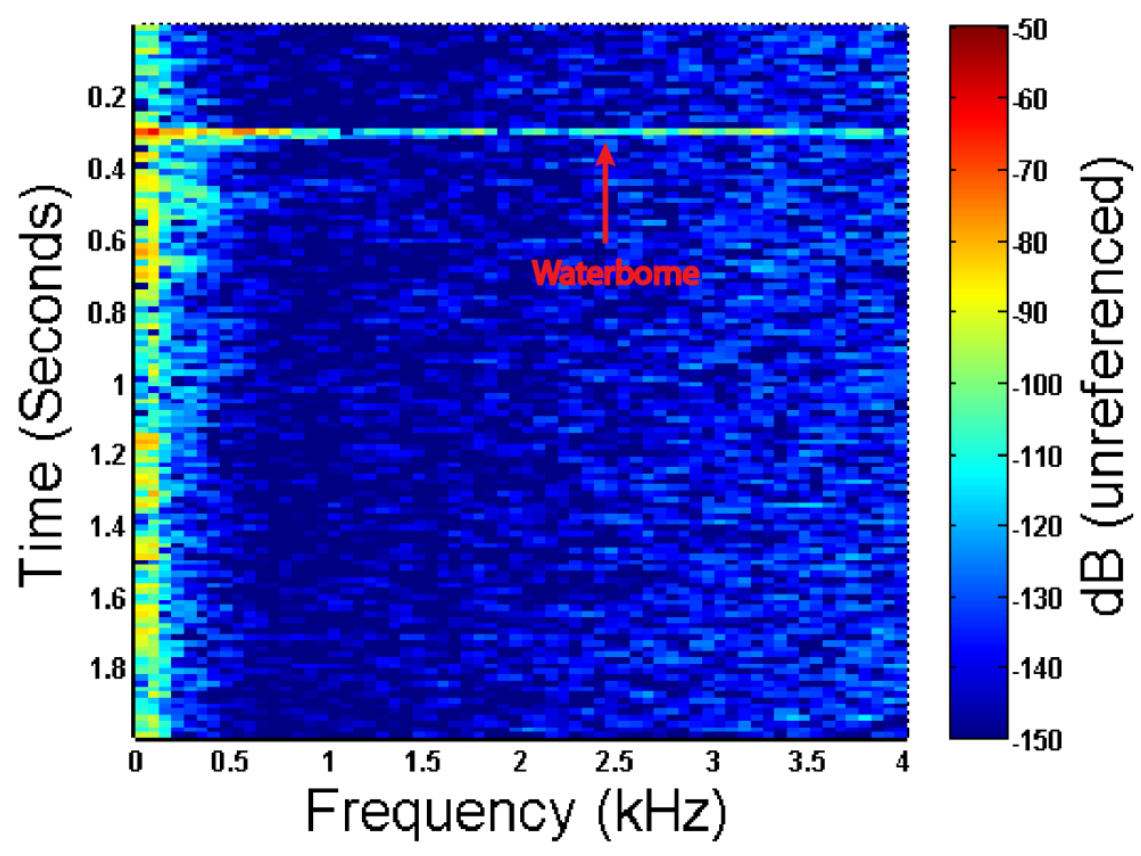

Figure 4.10 - Observed spectrogram of time series for sea ice deformation event one in Figure 4.9. 


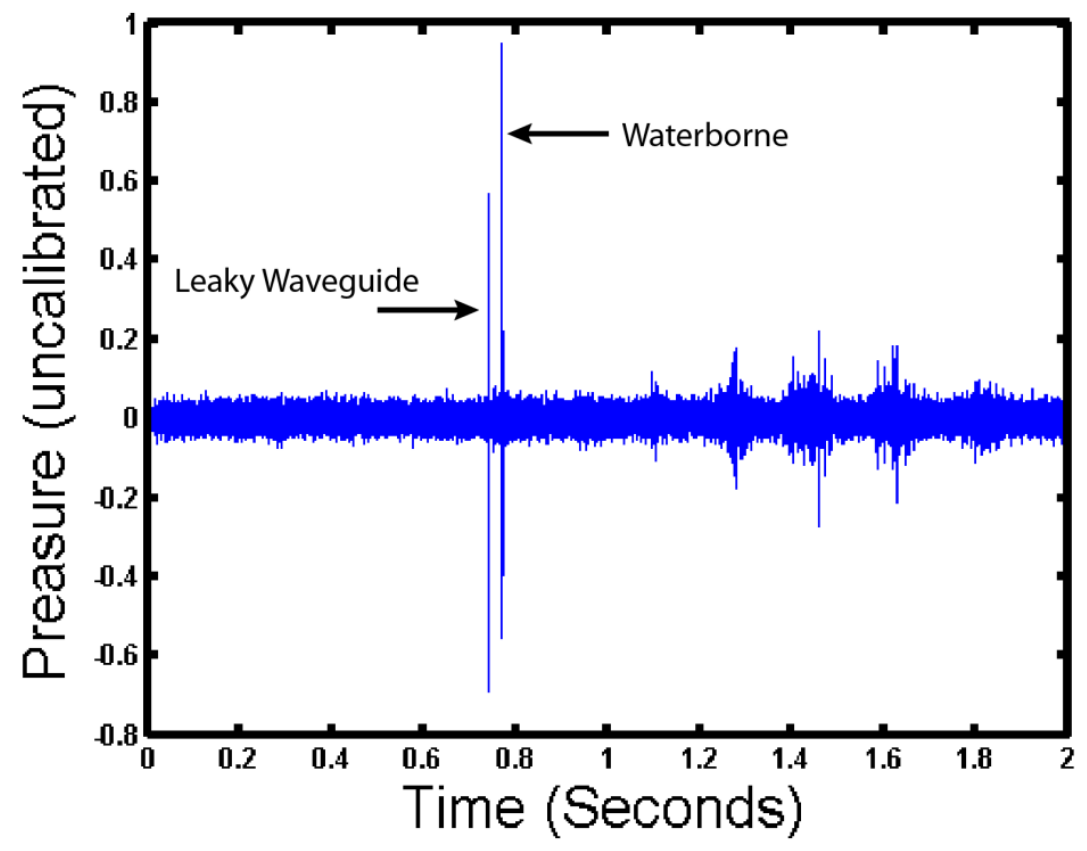

Figure 4.11 - Observed time series of sea ice deformation event two.

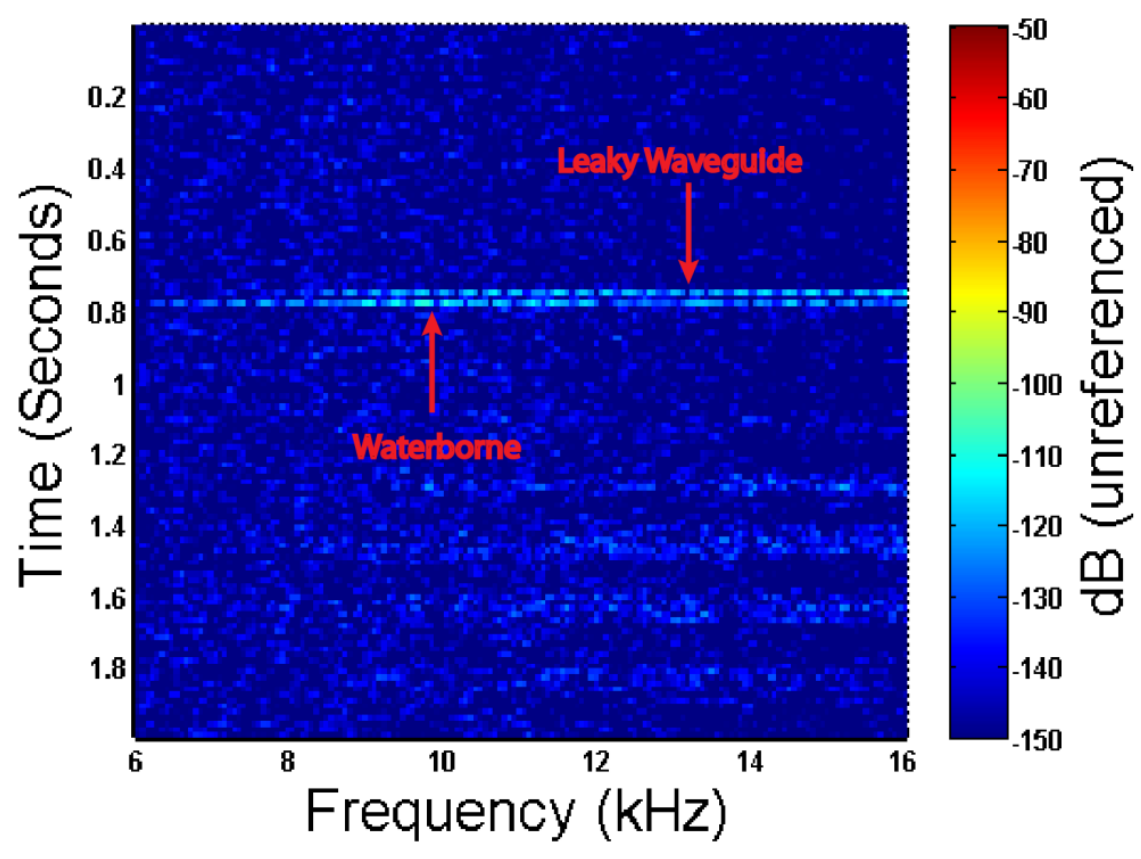

Figure 4.12 - Observed spectrogram of time series for sea ice deformation event one in Figure 4.11. 
The second deformation event exhibits a rapid duration event in the time series, Figure 4.11, along with leaky waveguide and waterborne characteristics in the spectrogram, Figure 4.12. The dominate characteristic of leaky waveguide arrivals being concentrated at the upper end of the frequency range is seen within the observed ambient noise data. A broadband frequency arrival quickly following the leaky waveguide arrivals was indicative of waterborne arrivals of the same event. These spectrogram characteristics were seen in the fractures that penetrated completely through the sea ice and fractures that were deep within the sea ice. 


\section{Chapter 5}

\section{Conclusion}

Fractures with higher velocity had smaller magnitudes for leaky waveguide arrivals and larger magnitudes for waterborne arrivals when compared to fractures with slower velocities. Tensile cracks that were deep within the sea ice had a more broadband frequency range for the waterborne arrivals, as more energy was transmitted through the sea ice to the water. However, waterborne arrivals for tensile cracks that were shallower within the sea ice had a lower frequency range that was less broadband when compared to the deeper tensile cracks as less energy was transmitted from the sea ice to the water. The waterborne arrivals for deeper fractures that had a more broadband range of frequencies in the spectrogram were accompanied by larger pressure magnitudes in the time series, when compared to the lower frequency less broadband shallower fractures, due to the increase in transmitted sound. The lowest frequency for leaky waveguide arrivals in all the simulations was above the calculated cutoff frequency of the leaky waveguide. This discrepancy is attributed to more interference taking place at lower frequencies. In contrast, the waterborne arrivals ranged from lower frequency bandwidths to more broadband frequency ranges - that encompassed the entire simulated range of frequencies - as the leaky waveguide formed by the sea ice water interface allowed the transmission of sound between the two media to take place at all frequencies.

The methods used to model Mode I fractures within sea ice appear to simulate observed ambient noise appropriately. Waveguide dynamics were readily seen in the simulated and observed data. Nevertheless, additions such as interference between sources, attenuation, shear within the ice and larger scale geometries need to be added to the methods for the model. These additions would increase the practicality of this model for simulating longer duration fracture 
events, and would even potentially extend the model's scope of application to additional types of fractures, structures and deformation. 


\section{References}

[1] R. J. Urick, "The Noise of Melting Icebergs," Journal of the Acoustical Society of America, pp. 337$341,1970$.

[2] N. C. Makris and I. Dyer, "Environmental correlates of Arctic ice-edge noise," Jounral of the Acoustical Societyt of America, vol. 90, no. 6, pp. 3288-3298, 1991.

[3] P. J. Stein, "Interpretation of a few ice event transients," Journal of the Acoustical Society of America, vol. 82, no. 2, pp. 617-621, 1988.

[4] O. I. Diachok and R. S. Winokur, "Spatial variablity of underwater ambient noise at the Artice-icewater boundary," Journal of the Acoustical Society of America, vol. 55, no. 4, pp. 750-753, 1974.

[5] T. C. Yang, C. W. Giellis and O. I. Diachok, "Acoustic properties of ice edge noise in the Greenland Sea," Journal of the Acoustical Society of America, vol. 82, no. 3, pp. 1034-1038, 1987.

[6] Y. Xie and D. M. Farmer, "Acoustical radiation from thermally stress sea ice," Journal of the Acoustical Society of America, vol. 89, no. 5, pp. 2215-2231, 1991.

[7] J. R. Fricke, "Acoustic scattering from elemental Arctic ice features: Numberical modeling results," Journal of the Acoustical Society of America, vol. 93, no. 4, pp. 1784-1796, 1993.

[8] J. R. Fricke and G. L. Unger, "Acoustic scattering from elemental Arctic ice features: Experimental results," Journal of the Acoustical Society of America, vol. 97, no. 1, pp. 192-198, 1995.

[9] D. M. Farmer and Y. Xie, "The sound generated by propagating cracks in sea ice," Journal of the Acoustical Society of America, vol. 85, no. 4, pp. 1489-1500, 1989.

[10] H. Schmidt, "OASES: Ocean Acoustic and Seismic Exploration Synthesis - Version 3.1 user guide and reference manual," Department of Ocean Engineering Massachusetts Institute of Technology, Cambridge, MA, 2011.

[11] M. Collins, "RAM: Range dependent Acoustic Model," Naval Research Laboratory, Washington, DC.

[12] M. Arakawa and V. F. Petrenko, "Observation of crack propagation in saline ice and freshwater ice with fluid inclusion," Canadian Journal of Physics, vol. 81, pp. 159-166, 2003.

[13] R. Gagnon, "High-speed video analysis of fracture propagation in a thick-edge-loaded fresh water ice sheet," Canadian Journal of Physics, vol. 81, pp. 261-269, 2003.

[14] F. V. Petrenko and O. Gluschenkov, "Crack velocities in freshwater and saline ice," Journal of 
Geophysical Research, vol. 101, pp. 11,541-11,551, 1996.

[15] Z. Li and Z. Bazant, "Acoustic Emissions in Fracturing Sea Ice Plate Simulated By Particle System," Journal of Engineering Mechanics, vol. 124, pp. 69-79, 1998.

[16] F. B. Jensen, W. A. Kuperman, M. B. Porter and H. Schmidt, Computational Ocean Acoustics, New York, NY: Springer, 2011. 